\title{
Fetal Hematopoiesis is Driven by Privileged Expansion and Differentiation of HSC-Independent Erythro-Myeloid Progenitors
}

\section{Laina Freyer}

Institut Pasteur

Lorea Iturri

Institut Pasteur

Anne Biton

Institut Pasteur

Alina Sommer

Institut Pasteur

Pascal Dardenne

Institut Pasteur

\section{Ana Cumano}

Institut Pasteur https://orcid.org/0000-0002-4578-959X

Elisa Gomez Perdiguero ( $\nabla$ elisa.gomez-perdiguero@pasteur.fr )

Institut Pasteur

\section{Article}

Keywords: developmental hematopoiesis, erythro-myeloid progenitors, hematopoietic stem cells, ontogeny, fetal liver, myeloid cells

Posted Date: October 28th, 2021

DOI: https://doi.org/10.21203/rs.3.rs-1002760/v1

License: (c) (1) This work is licensed under a Creative Commons Attribution 4.0 International License. Read Full License 


\title{
Fetal Hematopoiesis is Driven by Privileged Expansion and Differentiation of HSC-Independent Erythro-Myeloid Progenitors
}

\author{
Laina Freyer ${ }^{1}$, Lorea Iturri ${ }^{1}$, Anne Biton ${ }^{2}$, Alina Sommer ${ }^{1,3}$, Pascal Dardenne $^{1}$, Ana Cumano ${ }^{4}$ and Elisa Gomez \\ Perdiguero $^{1}$ \\ ${ }^{1}$ Institut Pasteur, Unit for Macrophages and Endothelial Cells, Developmental and Stem Cell Biology Department, UMR3738 \\ CNRS, 75015 Paris, France \\ ${ }^{2} \mathrm{Hub}$ de Bioinformatique et Biostatistique - Département Biologie Computationnelle, Institut Pasteur, 75015 Paris, France \\ ${ }^{3}$ Sorbonne Université, Collège Doctoral, F-75005 Paris, France. \\ ${ }^{4}$ Institut Pasteur, Unit for Lymphopoiesis, Immunology Department, INSERM U1223, 75015 Paris, France.
}

Correspondence: elisa.gomez-perdiguero@pasteur.fr

\section{Highlights}

- HSC contribute minimally to fetal myelopoiesis

- An 'EMP-to-HSC origin' switch in myelopoiesis occurs late in gestation

- Fetal liver GMP and MEP originate predominantly from yolk sac-derived EMP

- $\quad$ scRNAseq reveals the EMP-derived myeloid landscape of the mouse fetal liver

- EMP- and HSC-derived GMP are functionally inequivalent

\section{Keywords}

developmental hematopoiesis; erythro-myeloid progenitors; hematopoietic stem cells; ontogeny; fetal liver; myeloid cells

\begin{abstract}
Most blood and immune cells are produced by Hematopoietic Stem Cells (HSC) throughout life. However, several tissue resident immune populations can only be generated from developmentally restricted progenitors. This questions to what extent fetal HSC differentiate in utero, implicating an essential role for HSC-independent progenitors in supporting embryonic viability and innate immunity in the perinatal period. Among them, Erythro-Myeloid Progenitors (EMP) emerge from the extraembryonic yolk sac prior to HSC and their progeny (resident macrophages and skin mast cells) persist in adulthood. Here, we showed that HSC contributed minimally to fetal myelopoiesis as we exposed a developmentally-restricted privilege for erythro-myeloid differentiation from EMP in the fetal liver. EMPderived myeloid progenitors displayed distinct molecular features and were functionally inequivalent to fetal HSC-derived counterparts. These findings inform future studies of HSC-dependent and HSCindependent hematopoiesis in view of neonatal immunity and pediatric blood disorders for which the cell of origin is poorly understood.
\end{abstract}




\section{Graphical Abstract}

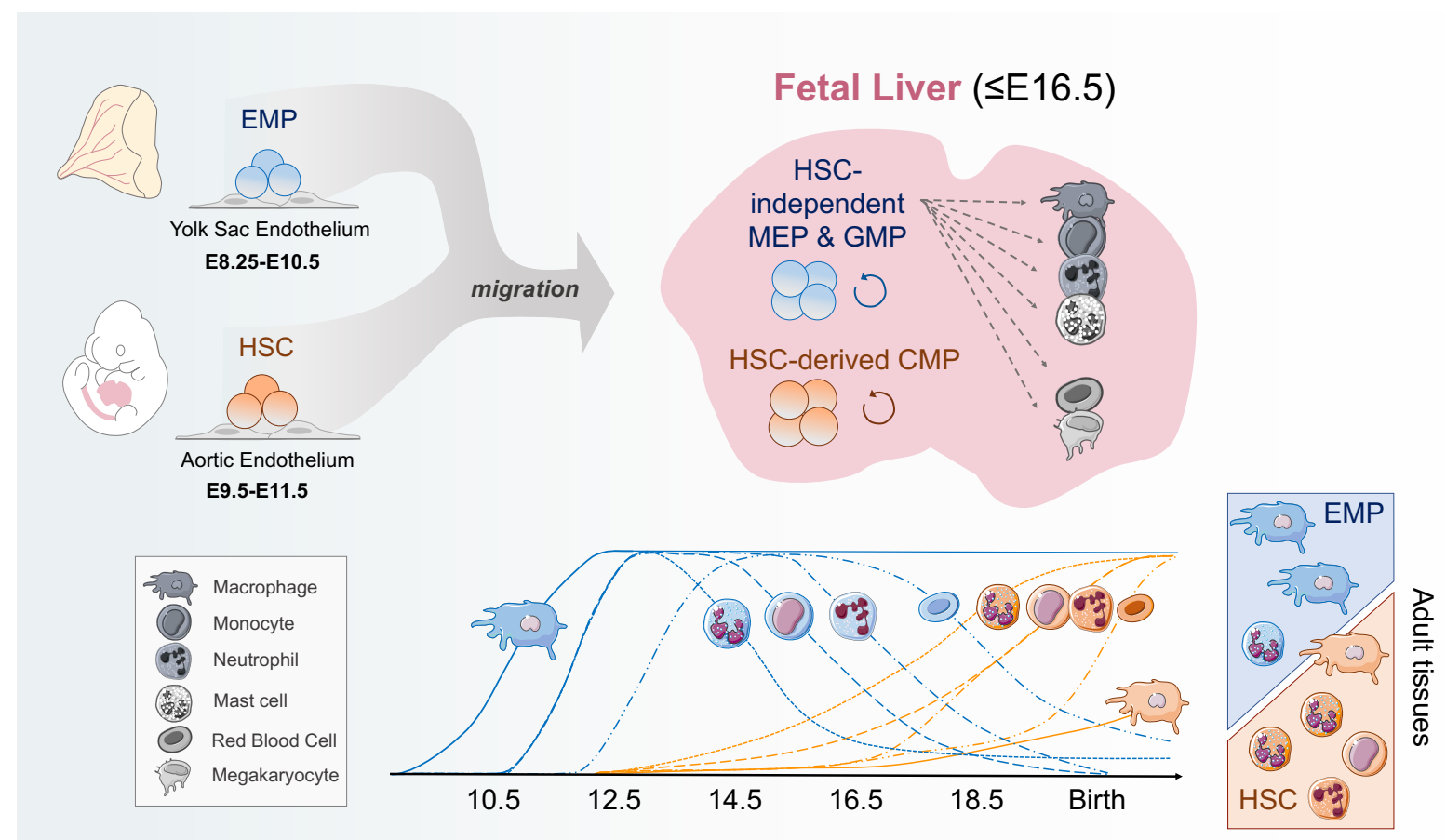




\section{Introduction}

The mammalian immune system develops in sequential waves or layers that have evolved to meet the needs of the growing embryo while simultaneously setting the groundwork for a lifetime of hematopoietic maintenance. The persistence of fetal-derived layers of tissue resident immune cells in adults (reviewed in Elsaid et al., 2020; Perdiguero and Geissmann, 2016) highlights the importance of exploring the link between cells that are functionally important in adult tissue homeostasis and disease but which are only produced during development (Bennett et al., 2018; Honold and Nahrendorf, 2018; Loyher et al., 2018; Zhu et al., 2017). The developmental hematopoietic system is a blend of blood and immune cells originating from at least two distinct waves of multipotent progenitors that overlap in space and time. The first wave, endowed with erythroid and myeloid potential and thus called EMP (ErythroMyeloid Progenitors), arise from the extraembryonic yolk sac between embryonic day (E)8.25 and E11.5 (Frame, 2015; Kasaai et al., 2017; Lux et al., 2008; Palis et al., 1999). They are responsible for red blood cells that support fetal survival (Chen et al., 2011; Soares-da-Silva et al., 2021) and adult tissue resident macrophages and mast cells that replenish independently of HSC (Ajami et al., 2011; Gentek et al., 2018; Ginhoux et al., 2010; Gomez Perdiguero et al., 2015; Hashimoto et al., 2013; Hoeffel et al., 2015; Schulz et al., 2012; Yona et al., 2013, 2013). The second wave of HSC (Hematopoietic Stem Cells) emerge intraembryonically (Ana Cumano et al., 1996; Cumano et al., 2001; Medvinsky and Dzierzak, 1996; Yvernogeau and Robin, 2017) from the aorta-gonad-mesonephros (AGM) region and other hemogenic sites between E10 and E12 (De Bruijn et al., 2000; Gekas et al., 2005; Ottersbach and Dzierzak, 2005). Unlike EMP, HSC give rise to lymphoid cells and are capable of long-term multilineage reconstitution. Yet, it remains unknown how the dynamic interplay between these overlapping waves, as they undergo expansion and differentiation side-by-side within the fetal liver niche, governs their unique functional roles in establishing the immune system.

In this study, we addressed the respective contribution of each progenitor wave to fetal hematopoiesis with a focus on functional characterization of EMP-derived hematopoiesis within the fetal liver. We found that HSC contributed minimally to macrophages, mast cells and blood circulating monocytes and neutrophils before birth. Rather, the major source of fetal liver myeloid cells was supplied by preferential amplification of EMP-derived erythroid and myeloid progenitor intermediates. We further explored EMP hematopoiesis on the molecular level using single-cell RNA sequencing (scRNAseq) coupled with expression of cell surface markers. scRNAseq revealed transcriptional heterogeneity of pulse chase labeled EMP-derived myeloid progenitors and showed that EMP myelopoiesis was biased towards production of mast cells and granulocytes in the fetal liver. Furthermore, EMP-derived myeloid progenitors retained extensive in vitro erythroid potential and combinatorial expression of lineage defining factors, thus highlighting molecular and functional differences with fetal HSC-derived counterparts. Finally, scRNAseq demonstrated that immunophenotyping of fetal myeloid progenitors based on paradigms of adult hematopoiesis was not sufficient to capture the molecular and functional diversity inherent to fetal liver hematopoietic progenitors with mixed ontogeny. 


\section{Results}

\section{Fetal erythroid and myeloid cells are mainly HSC-independent.}

Inherent to EMP and HSC development are their endothelial-to-hematopoietic origins meaning that genetic lineage tracing approaches are hindered by the extent of common nuclear and cell surface markers. Pulse chase labeling has been indispensable for tracing EMP independently of HSC using Csf1rMericreMer mice (Gomez Perdiguero et al., 2015; Schulz et al., 2012). Recently, Cdh5 ${ }^{\text {CreERT2 mice }}$ have been used to take advantage of spatiotemporal differences in EMP versus HSC origins from distinct hemogenic endothelia (Gentek et al., 2018; Simic et al., 2020).

To measure the in vivo contribution of fetal HSC to mature hematopoietic lineages (neutrophils, $\mathrm{Nt}$; monocytes, MO; mast cells, Mast; macrophages, MF; red blood cells, RBC; and megakaryocytes, Mk), we performed flow cytometry analysis of cells from pulse chase labeled Cdh5 ${ }^{\text {CreERT2 }}$ Rosa26 $^{\text {YFP }}$ embryos (Fig. 1, Extended Data Fig. 1a). HSC (LSK, Lin ${ }^{\text {neg }}$ Sca $^{+} \mathrm{Kit}^{+}$) were selectively labeled by injection of 4-hydroxytamoxifen (OHT) at E10.5, whereas EMP were labeled by injection of OHT at E7.5 with the caveat that this also labeled few LSK (Extended Data Fig. 1b). However, LSK labeled by E7.5 OHT did not contribute significantly to long-term adult hematopoiesis (Extended Data Fig. 1c). To control for inherent variabilities between individual pulse chase labeling manipulations and to take into account the labelling of LSK by both injection timepoints, the percentage of YFP among mature cells was normalized to the percentage of YFP among microglia $\left(C D 45^{+}\right.$Kit $\left.^{\text {neg }} \mathrm{F} 4 / 80^{\text {hi }} \mathrm{CD} 11 \mathrm{~b}^{+}\right)$for E7.5 OHT injections or to the percentage of YFP among LSK for E10.5 OHT injections (Fig. 1a).

We observed an EMP-to-HSC switch in cellular origin around E16 for monocytes (CD45 ${ }^{+}$Kit $^{\text {neg }}$ F4/80 ${ }^{\text {lo }}$ CD11 $\mathrm{b}^{+}$CSF1 $\mathrm{R}^{+} \mathrm{Ly}_{6 \mathrm{C}^{+/-}}$) and mast cells (Lin ${ }^{\text {neg }}$ Sca1 ${ }^{\text {neg }} \mathrm{Kit}^{+} \mathrm{Itgb}^{+} \mathrm{CD} 16 / 32^{\mathrm{hi}}$ ) (Hamey et al., 2020) then $48 \mathrm{~h}$ later, at $\mathrm{E} 18$, for neutrophils $\left(C D 45^{+} \mathrm{Kit}^{\text {neg }} \mathrm{CSF} 1 \mathrm{R}^{\text {neg }} \mathrm{CD} 11 \mathrm{~b}^{+} \mathrm{Ly} \mathrm{G}^{+}\right)$(Fig. 1a). This was also observed for erythroid lineages (Extended Data Fig. 1d-e) (Iturri et al., 2021; Soares-Da-Silva et al., 2021). As expected, labeling efficiency of macrophages (CD45 ${ }^{+} \mathrm{Kit}^{\text {teg }} \mathrm{F} 4 / 80^{\text {hi }} \mathrm{CD} 11 \mathrm{~b}^{+}$) paralleled labeling of the microglia after E7.5 OHT injection (Fig. 1a), validating the predominant EMP origin of tissue macrophages as described previously (Gomez Perdiguero et al., 2015). Neutrophils and macrophages were the most abundant myeloid cells in the fetal liver (Fig. 1b) for which the contribution of HSC was only evident at E18.5 (Fig. 1c). In the case of tissue macrophages, the input from HSC was insignificant considering the total number of macrophages per fetal liver (Fig. 1b-c). In peripheral blood, the relative numbers of circulating monocytes, neutrophils and mast cells exhibited distinct temporal abundance (Fig. 1d) and were mostly EMP-derived (Fig. 1e). Circulating HSC-derived monocytes and neutrophils were only observed in blood just before birth (Fig. 1e). Interestingly, EMP-derived neutrophils were generated in the fetal liver as early as E12.5 but only released into circulation between E14.5-16.5, in contrast to EMP-derived monocytes and mast cells that were detected at the same time in fetal liver and blood (Fig. 1c,e). Taken altogether, most mature erythroid and myeloid cells were generated by EMP during development while HSC myelopoiesis generated late waves of fetal monocytes, mast cells and neutrophils, each with their own differentiation dynamics. 


\section{Delayed commitment of fetal HSC to GMP and MEP.}

The deficiency in mature erythroid and myeloid cells produced from fetal HSC prompted us to examine the ontogeny of erythro-myeloid commitment at the progenitor level (Fig. 2). HSC-derived myeloid progenitors are classified by a hierarchy of progenitor states based on canonical immunophenotypes (cell surface expression of CD16/32 and CD34). A common myeloid progenitor (CMP) gives rise to further committed megakaryocyte-erythroid progenitors (MEP) and granulocyte-monocyte progenitors (GMP) (Akashi et al., 2000). This hierarchy is phenotypically and functionally conserved in the E14.5 fetal liver (Traver et al., 2001) except for notable differences in colony-forming burst activity.

Using the paradigm of CMP-to-GMP/MEP commitment, we examined cell surface of expression of CD16/32 and CD34 among E12.5-18.5 fetal liver hematopoietic progenitors (LK, Lin ${ }^{\text {neg }} \mathrm{Kit}^{+}$Sca1 ${ }^{\text {neg }}$ ) labeled by Cdh5 $5^{\text {CreERT2 Rosa26 }}{ }^{\text {YFP }}$ (Fig. 2a). Among total LK, the frequency of CMP, GMP and MEP was stable from E14.5 onwards, with MEP representing the majority of LK progenitors ( $78 \% \pm 0.4 \mathrm{sem}$ ) (Fig. 2b). HSC-derived progenitors were predominantly CMP at E12.5, with HSC-derived GMP undetected at this stage, while the frequency of HSC-derived MEP increased over time (Fig. 2b). Indeed, fetal liver GMP and MEP were largely devoid of HSC progeny until E16.5 (Fig. 2c), despite the rapid expansion of these populations with time (Extended Data Fig. 2a). Instead, GMP and MEP were populated by EMP progeny between E12.5 and E16 (Fig. 2c), highlighted by the contrasting number of $\mathrm{YFP}^{+}$progenitors per fetal liver that were labeled by E7.5 OHT injection compared to E10.5 injection (Fig. 2d). Conversely, the labeling of CMP by E7.5 OHT did not exceed equilibration with LSK (Extended Data Fig. 2b), supporting the observation that CMP were almost exclusively HSC-derived from E12.5 onwards. Altogether, although HSC seed the fetal liver from E10.5 onwards and HSC-derived CMP were present in the fetal liver as early at E12.5, the progression of HSC towards more committed progenitors (such as GMP and MEP) was delayed until E16.5.

\section{Transit amplification of EMP-derived intermediates.}

We next to sought to investigate how short-lived multipotent progenitors such as EMP, that are generated between E8.5 and E11.5, could sustain erythro-myelopoiesis throughout most of fetal development. In the fetal liver, the fold change in expansion of EMP-derived intermediates (GMP and MEP) exceeded HSC-derived progenitors from E12.5 to E16.5 (Extended Data Fig. 2c), even though the total number of LSK were also expanding during this time (Extended Data Fig. 2d). To compare proliferative potential between EMP-derived and HSC-derived myeloid progenitors (CMP and GMP), we performed single-cell colony forming unit assays (CFU) from fetal liver at E11.5 (predominantly EMPderived) and E18.5 (predominantly HSC-derived) (Fig. 2e, Extended Data Fig. 2e). While colony forming efficiency was similar between EMP- and HSC-derived myeloid progenitors (Extended Data Fig. 2e), there was a striking difference in colony size; E11.5 EMP-derived myeloid progenitors gave rise to colonies with 2.6-fold more cells on average compared to E18.5 HSC-derived counterparts when cultured with saturating levels of SCF (Kit Ligand) (Fig. 2e). Expansion of EMP-derived progenitors was moderately affected by decreasing levels of SCF, and indeed E11.5 EMP-derived progenitors outperformed HSC-derived progenitors at all SCF concentrations (Fig. 2e). 
We thus investigated the cell cycle status of EMP-derived progenitors in situ and showed that $50 \%$ of E11.5 GMP were in G2M, twice what was observed for CMP and MEP (Fig. 2f). This amplification at the GMP level preferentially occurred locally in the fetal liver niche, as opposed to amplification of progenitors in the peripheral circulation (Fig. 2f). Finally, we performed adoptive transfer of E11.5 EMP-derived myeloid progenitors into sub-lethally irradiated $\mathrm{Rag}^{-/} \mathrm{gc}^{-/}$newborns (Extended Data Fig. 2f). Finally, we performed in vivo adoptive transfer of E11.5 and E18.5 fetal liver myeloid progenitors (CMP and GMP) from Rosa26 $6^{m T m G}$ donors into sub-lethally irradiated Rag $^{-/} \mathrm{gc}^{-/}$newborns (Extended Data Fig. 2f). After 5 days, EMP-derived cells (mTomato ${ }^{+}$cells from E11.5 donors)

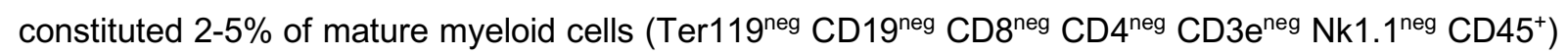
in the bone marrow, liver and blood of recipient mice (Extended Data Fig. 2f). However, HSC-derived cells (mTomato ${ }^{+}$cells from E18.5 donors) were barely detectable 5 days after transfer and required an additional 48 hours to produce mature myeloid cells (Extended Data Fig. 2f). Collectively, these findings suggest that permissive conditions in the fetal liver favored transit amplification of EMP-derived GMP and MEP which underwent rapid expansion and differentiation compared to HSC-derived progenitors under the same conditions in vitro and in vivo.

\section{EMP-derived myeloid landscape revealed by scRNAseq.}

The predominance of EMP-derived myeloid cells in the early fetal liver led us to investigate the lineageand ontogeny-specific characteristics of EMP differentiation at the molecular level. To focus on EMPderived progenitors at early stages (E10.5 to E14.5), we used the Csf1rmericreMer Rosa26 ${ }^{\text {YFP }}$ model that reliably marks newly emerged EMP independently of LSK (Extended Data Fig. 3a-b). Fetal liver LK $\left(\mathrm{Lin}^{\text {neg }} \mathrm{Kit}^{+} \mathrm{Sca}{ }^{\text {neg }}\right)$ were pulse-labeled by injection of OHT at E8.5 or E9.5 then characterized by flow cytometry to assess the contributions of EMP to early CMP, MEP and GMP populations (Fig. 3a-b). For comparability between E8.5 and E9.5 OHT injection experiments, we normalized the percentage of YFP among EMP-derived progenitors to the labeling of megakaryocytes (Mk, Kit $\left.{ }^{\text {neg }} \mathrm{CD} 45^{\text {neg }} \mathrm{CD} 41^{\mathrm{hi}}\right)$ that are EMP-derived at these stages (Iturri et al., 2021). In line with results from Cdh5 CreERT2 Rosa26 ${ }^{\text {YFP }}$ fate mapping (Fig. 2c-d), EMP were the main contributor to GMP and MEP until E14.5 (Fig. 3a). Further, EMP-derived CMP were present until E11.5 when pulse-labelling with E9.5 OHT, but declined rapidly thereafter, confirming that most CMP are HSC-derived from E12.5 onwards. Finally, separation of OHT injection timepoints by 24 hours linked the 'time of birth' of EMP in the yolk sac to the time at which they peak among GMP and MEP. The numbers of EMP-derived GMP and MEP peaked three days after induction by both OHT pulses (Fig. 3b), demonstrating that EMP commitment is either intrinsically regulated or requires the same length of exposure time within the fetal liver niche for their expansion.

Since two-dimensional analysis of cell surface markers (CD16/32 and CD34) could not fully capture the heterogeneity of EMP-derived CMP, MEP and GMP, we performed FACS-based massively parallel single-cell RNA sequencing (MARS-seq) (Jaitin et al., 2014; Keren-Shaul et al., 2019) that utilizes index sorting for retrospective analysis of cell surface markers corresponding to individual cellular transcriptomes. EMP-derived LK were isolated from Csf1 $r^{\text {Mericremer }}$ Rosa26 ${ }^{\text {YFP }}$ embryos pulsed with E8.5 OHT injection and 1,590 cells from E10.5 fetal liver and 1,405 cells from E12.5 fetal liver were analyzed (Extended Fig. 3c). UMAP representation of clustered cells demonstrated two major pan- 
myeloid (clusters 2-5) and pan-erythroid (clusters 6-10) branches bridged by a cluster of putative EMP (cluster 1) (Extended Fig. 3c). Annotation of cell types was guided by pairwise correlation with E14 fetal liver hematopoietic cells from the Mouse Cell Atlas (Han et al., 2018) (Extended Data Fig. 3d). The EMP cluster correlated most closely with stem/progenitor cells while additional myeloid clusters were identified as GP (Granulocyte Progenitors, highly correlated with Elane ${ }^{\text {hi }}$ neutrophils) and mast cells. The EMP cluster was characterized by combinatorial erythroid and myeloid gene expression with few cluster-specific markers (Extended Data Fig. 3e) and a high proportion of cells assigned to the G2M phase of the cell cycle (Extended Data Fig. 3f). To extend our analysis of myeloid cells, we integrated our scRNAseq data with two existing datasets (Fig. 3c-d). This included CD45 EMP, pre-macrophages and macrophages from E10.25 embryos (Mass et al., 2016) as clusters 11-13, and EMP-derived fetal liver myeloid cells from E14.5 Csf1 $r^{\text {MericreMer }}$ Rosa26 $6^{\text {tdTomato }}$ embryos pulse-labeled with E8.5 OHT injection (Yahara et al., 2020) as clusters 14-19 (Fig. 3c-d). The three-way integration between myeloid progenitors and mature cells confirmed that EMP-derived myeloid progenitors in the fetal liver were biased towards granulocyte and mast cell lineages (Fig. 3c-d).

Collectively, these analyses showed that at the onset of fetal liver colonisation, EMP-derived progenitors seeding the fetal liver were mostly comprised of multipotent progenitors (EMP, cluster 1) and early myeloid, erythroid and Mk progenitors (clusters 2, 6, 7 and 10, respectively) that started their differentiation in the yolk sac niche (Iturri et al, 2021). EMP-derived progenitors subsequently transited towards more committed states, with granulocyte progenitors (GP, cluster 3) and mast cell progenitors (Mast, cluster 5) identified at E12.5. The underrepresentation of Kit ${ }^{+}$macrophage progenitors in the E12.5 fetal liver was likely due to their earlier differentiation in the yolk sac that precedes trafficking of pre-macrophages (Stremmel et al., 2018). Kit ${ }^{\text {neg }} \mathrm{F} 4 / 80^{+}$pre-macrophages can self-propagate within target tissues between E10.5-E12.5 and were captured by the Mass et al. dataset as CD45 cells from E10.25 embryos that linked the trajectory from EMP to mature tissue macrophages (Fig. 3d).

\section{HSC-derived and EMP-derived GMP are functionally inequivalent.}

To probe whether EMP-derived and HSC-derived GMP have different properties, we examined single cell transcriptomes and performed clonal functional assays (Fig. 4, Extended Data Fig. 4). FACS-based scRNAseq allowed us to retrospectively examine cell surface expression of CD16/32 and CD34, revealing immunophenotypic heterogeneity of transcriptionally profiled EMP-derived progenitors (Fig. 4a). Most cells in myeloid clusters (MP and GP) displayed a CMP or GMP immunophenotype at E10.5 and E12.5, with $51 \%$ of E12.5 GP cells plotted as GMP (Extended Data Fig. 4a). Conversely, quantification of scRNAseq cluster identities among canonically gated populations confirmed that GMP were enriched for myeloid progenitors, when compared to CMP and MEP (Fig. 4b). However, erythroid clusters unexpectedly constituted $\sim 25 \%$ of immunophenotypic GMP (Fig. 4b), thus highlighting transcriptional heterogeneity at the population level.

Surprisingly, this transcriptional heterogeneity was also evident at the single cell level among committed myeloid progenitors (GP and Mast) that exhibited persistent co-expression of lineage defining transcription factor genes KIf1 (red blood cells) and Cebpe (neutrophils) or Gata2 (mast cells) among individual myeloid progenitors (Fig. 4c-d). For example, 46\% of cells in the GP cluster co-expressed 
KIf1 and Cebpe (Fig. 4d), which was not observed in HSC-derived granulocyte progenitors that were identified by scRNAseq from the adult bone marrow (Extended Data Fig. 4b-c) (Paul et al., 2015). To determine whether this reflected functional heterogeneity, we sorted CMP and GMP from E11.5 and E18.5 fetal liver (representing EMP and HSC waves respectively) and performed single cell in vitro colony-forming unit (CFU) assays (Fig. 4e, Extended Data Fig. 4d-f). Similar to our observations from single cell transcriptomic profiling, E11.5 fetal liver GMP demonstrated significant in vitro erythroid (RBC or RBC/Mk) functional potential when compared to E18.5 GMP (Fig. 4e). More than 50\% of GMP colonies contained erythroid cells, doubling the predicted $25 \%$ erythroid progenitors among GMP that were identified by scRNAseq (Fig $\mathbf{4 b}$ ). Further, CFU assays revealed that the heterogeneity in lineage potential is greater among EMP-derived progenitors, regardless of their immunophenotype, as evidenced by the substantial erythroid and mixed output of EMP-derived CMP and GMP. This may be a result of differences in the accessibility of the transcriptional landscape in EMP-derived myeloid progenitors compared to HSC counterparts, or it may imply that classical immunophenotypic gating strategies poorly capture the functional diversity of EMP-derived progenitors at early stages.

\section{DISCUSSION}

Fetal and neonatal myeloid cells are distinct from adult HSC-derived myeloid cells in their expression of Ig receptor complexes, antigen-presenting molecules, co-expression of lymphoid genes and production of polarizing cytokines (Elsaid et al., 2021; Gentek et al., 2018; Krow-Lucal et al., 2014; Levy, 2007). Distinct developmental origins of fetal/neonatal myeloid cells from EMP or fetal HSC respectively may inform some of these differences. This is because during development, the fetal liver is the prime hematopoietic organ, supporting differentiation from at least two distinct pools of hematopoietic progenitors with unique extra-embryonic (EMP) versus intra-embryonic (HSC) origins. Our study has pinpointed a 'switch' from EMP-to-HSC origin in fetal liver erythro-myelopoiesis that occurs around E15 for progenitors and E16 for mature cells. Investigation into the allocation of EMP and HSC to transit amplifying progenitor subsets showed that EMP rapidly generate GMP and MEP while HSC are slower in this process. One possibility is that this may be due to spatiotemporally distinct requirements for growth factors and cytokines. For example, Soares-da-Silva and colleagues have shown that EMPderived erythroid progenitors express high levels of Epor thereby endowing them with an advantage over HSC in sensing the availability of EPO in the fetal liver and driving EMP-derived erythropoiesis (Soares-da-Silva et al., 2021). We could imagine a similar scenario for myelopoiesis, however testing this is complicated by the diversity of myeloid lineages that can be generated by a larger combination of cytokines. In this regard, our findings that EMP-derived GMP outperform HSC-derived GMP in low SCF conditions support the hypothesis that EMP-derived myeloid progenitors can still flourish in the fetal liver with low levels of SCF compared to HSC-derived myeloid progenitors. This is in line with a previous report which demonstrated that early YS and FL hematopoiesis (before E12.5) is less dependent on Kit-SCF signaling based on in utero injection of a Kit blocking antibody (Ogawa et al., 1993). 
Using two independent genetic pulse chase labeling models to lineage trace the origins of mature cells from EMP or HSC, we found that the contribution of HSC to mature myeloid cells in the fetal liver is gradual and that EMP carry the burden of erythroid and myeloid production. This may be a mechanism to ensure embryonic survival following the first wave of primitive red blood cells since fetal HSC are incapable of providing sufficient red blood cells (Chen et al., 2011). Interestingly, EMP-derived progenitors retain substantial erythroid potential after committing to myeloid lineages, both at the population and single cell level, when compared to HSC counterparts. Furthermore, EMP are the primary source of fetal tissue resident (macrophages and mast cells) and circulating myeloid cells (neutrophils). The functional requirements for fetal myeloid cells in developmental processes remains elusive and is a topic of current investigations. However, it is clear that fetal myeloid cells are essential for survival after birth as mice that are null for Pu.1 die rapidly from septicemia (McKercher et al., 1996) and efficient recruitment of neutrophils is essential for bacterial clearance in the neonatal period (Andrade et al., 2013). Therefore, establishing the fetal myeloid innate immune system is critical to compensate for immaturity of newborn adaptive immunity that is evident at birth (Levy, 2007). By laying the burden of fetal erythro-myelopoiesis on EMP, there is little demand for fetal HSC output. This may provide opportunity for HSC to expand and mature within the fetal liver prior to seeding the bone marrow.

Collectively, our findings support the notion that the developing fetal hematopoietic system uses a layered approach in order to supply sufficient innate immune and blood cells that are not only necessary to sustain survival of the fetus (Chen et al., 2011; Soares-da-Silva et al., 2021) but are also critical for organogenesis (Elsaid et al., 2021; Simic et al., 2020) and priming of protective immunity (Msallam et al., 2020). While the time of residence of EMP and HSC waves overlaps in the fetal liver, it is apparent that EMP carry the burden of erythro-myelopoiesis while HSC undergo expansion and to some extent innate lymphopoiesis from transient fetal HSC (Elsaid et al., 2021; Simic et al., 2020). It remains to be determined how the execution of these developmental hematopoietic programs is regulated by both intrinsic (ontogeny) and extrinsic (niche) factors that are spatiotemporally restricted to embryogenesis.

\section{ACKNOWLEDGEMENTS}

We thank Baptiste Saudemont and Yann Loe-Mie for help with the MARS-Seq pipeline, Rebecca Gentek, Marc Bajénoff and Kémy Ade for exploratory Cdh5CreERT2 experiments, Yvan Lallemand for handling and injections of mice, Sébastien Bastide and Caroline Kaiser for advice on scRNAseq analysis, Laure Bally-Cuif for critical reading of the manuscript, Antonio Bandeira Ferreira for neonatal injections and other members of the Gomez Perdiguero and Cumano groups for critical discussions and feedback. We appreciate the support of the UTECHS CB, C3BI and Animalerie Centrale platforms. This work was funded by the Institut Pasteur, the CNRS, Revive (Investissement d'Avenir; ANR-10-LABX73) and the European Research Council ERC investigator award (2016-StG-715320 to E.G.P.) E.G.P. also acknowledges financial support from the Fondation Schlumberger (FRM FSER 2017) and the Emergence(s) program from Ville de Paris (2016 DAE 190). L.I. was supported by a PhD fellowship from the Revive Labex and L.F. by a Florence Gould-Pasteur Foundation fellowship. 


\section{Methods}

\section{Mice}

All mice used in this study have been previously described. Experimental procedures, housing and husbandry were in compliance with the regulatory guidelines of the Institut Pasteur Committee for Ethics and Animal Experimentation (CETEA, dap160091). Strains included Csf1r MericreMer transgenic (FVB background, MGI:5433683), Rosa26 ${ }^{\text {YFP }}$ (C57BI6 background, MGI:2449038), Cdh5 ${ }^{\text {CreERT2 }}$ transgenic (C57BI6 background, MGI:3848982), Rag2-/ ; $\mathrm{YC}^{-/-}$CD45.1/1 and Rosa26 ${ }^{m T m G}$ mice. Timed matings were performed and the date of vaginal plug was considered E0.5. Embryonic stage was validated using morphological landmarks. Genotyping procedures are available upon request.

\section{OHT Preparation and Injection}

4-hydroxytamoxifen (OHT) (Sigma, H7904-25MG) was dissolved in equal parts of ethanol and Kolliphor (Sigma C5135-500G) using sonication. 10mg/mL stocks of progesterone (P3972-5G) were prepared by resuspending in ethanol and sunflower oil (Sigma S5007-250ML). For pulse-labeling, OHT was coinjected with progesterone to reduce the risk of abortion. For pulse-labeling using the Csf1rMericreMer strain, females were weighed on day 8 of pregnancy and injected with $75 \mu \mathrm{g} / \mathrm{g}$ (body weight) OHT and $37.5 \mu \mathrm{g} / \mathrm{g}$ (body weight) progesterone. For pulse-labeling using the Cdh5 ${ }^{\mathrm{CreERT2}}$ strain, females were

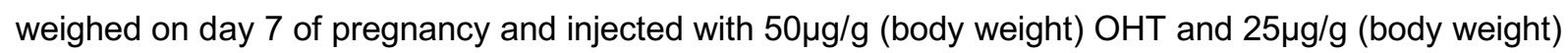
progesterone. Live births of pulse-labeled mice were complicated by OHT injection during pregnancy, therefore we performed caesarean sectioning followed by fostering with FVB females.

\section{Flow Cytometry}

Pregnant mice were killed by cervical dislocation and embryos were dissected in cold PBS. Fetal peripheral blood was collected in 2mM EDTA by severing the vitelline and umbilical vessels after removing the placenta and extraembryonic membranes. Organs were enzymatically dissociated in digestion buffer composed of PBS with 1mg/mL collagenase D (Sigma 11088882001), $100 \mathrm{U} / \mathrm{mL}$ DNasel (DN25-100mg) and 3\% Fetal Bovine Serum. Cells were passed through 100 $\mu$ m strainers by mashing with the piston of a $5 \mathrm{~mL}$ syringe and then collected in cold filtered FACS Buffer $(0.5 \%$ BSA and $2 \mathrm{mM}$ EDTA in PBS). For washing, cells were centrifuged at $320 \mathrm{~g}$ for 7 minutes. Blocking was performed with 5\% FBS and 1:20 Mouse IgG (Interchim 015-000-003) in FACS Buffer followed by 30 minutes of antibody staining. Cells were washed and incubated with fluorescently-conjugated streptavidin for 20 minutes. For experiments with adult mice, $2.4 \mathrm{mg}$ Dispase was added to the digestion buffer and tissue was minced with scissors before digestion. Blood was collected by retro-orbital bleeding. Red blood cell lysis was performed on all organs one time and twice for blood. Bone marrow was flushed from the femur and tibia using a $25 \mathrm{G}$ needle. Stained cells were passed on the BD Symphony A5 cytometer (FACSDiva v7.0 software) or Cytoflex LX (CytExpert v2.1 software). To quantify cells, the following formula was used : (\# of cells acquired) $x$ (volume of resuspended cells after staining and washing/volume of cells acquired) $x$ (volume of cell suspension in blocking buffer prior to staining/volume of cells plated for staining). Results were analyzed and plots generated using FlowJo v10 software. 


\section{Intracellular Staining}

To assay cell cycle, cells were collected for flow cytometry and stained for cell surface proteins as described above. Following cell surface staining, cells were fixed in $4 \%$ paraformaldehyde for 15 minutes on ice, washed, then permeabilized in $0.1 \%$ saponin with $2 \%$ normal goat serum for 15 minutes on ice. Cells were incubated with intracellular markers (Ki67, 1:50; DAPI, 1:1000) in $0.1 \%$ saponin for 1 hour on ice, washed in $0.1 \%$ saponin then resuspended in FACS buffer. Stained cells were passed on the BD Symphony A5 cytometer and DAPI was recorded in linear.

\section{Antibodies}

\begin{tabular}{|c|c|c|}
\hline Antigen & Conjugates & Clone \\
\hline CD16/32 & BUV737 & $2.4 \mathrm{G} 2$ \\
\hline CD45 & BUV661, BUV395 & $30-F 11$ \\
\hline CD45.2 & APC-Cy7 & 104 \\
\hline CD41 & BUV395 & MWReg30 \\
\hline $\mathrm{F} 4 / 80$ & BV421, BV785, biotin & BM8 \\
\hline Ly6G & BV711 & 1A8 \\
\hline Ly6C & BV605 & HK1.4 \\
\hline Sca1 & BV510, BV711 & D7 \\
\hline CD34 & eF450 & RAM34 \\
\hline Itgb7 & PE-Cy7 & FIB504 \\
\hline Ter119 & biotin & TER-119 \\
\hline CD19 & biotin & 1D3 \\
\hline CD3e & biotin & $145-2 \mathrm{C} 11$ \\
\hline Gr1 & biotin & RB6-8C5 \\
\hline NK1.1 & biotin & PK136 \\
\hline CD115 & APC, PE-CF594 & AFS98 \\
\hline Flt3 & PE, PerCP-eF710 & A2F10 \\
\hline Kit & APC-Сy7, PE & 2B8 \\
\hline CD11b & AF700, PE-Cy7 & $\mathrm{M} 1 / 70$ \\
\hline AA4.1 & APC, PE-CF594 & AA4.1 \\
\hline CD61 & PE-Cy7 & 2C9.G2 \\
\hline CD71 & AlexaFluor647 & $\mathrm{C} 2$ \\
\hline CD150 & PE-Cy7 & mShad150 \\
\hline CD48 & APC & HM48-1 \\
\hline Ki67 & $\mathrm{PE}$ & B56 \\
\hline SAV & PE-Cy5, BUV737 & \\
\hline
\end{tabular}

\section{Single-Cell Library Preparation}

Cells were prepared for fluorescence-activated cell sorting (FACS) as described for flow cytometry. Embryos were screened for YFP expression by rapidly dissociating the head with a syringe, filtering, then passing unstained cells on the Beckman Coulter Cytoflex cytometer. YFP ${ }^{+}$embryos were pooled. 
Lineage cells (Ter119+ $\mathrm{F} 4 / 80^{+} \mathrm{Gr} 1^{+}$) were depleted using magnetic anti-biotin Microbeads (1:5) (Miltenyi Biotec 130-090-485) and MS columns (Miltenyi Biotec 130-042-201). Lin ${ }^{\text {neg }} \mathrm{Kit}^{+} \mathrm{YFP}^{+}$cells were index sorted using a FACSAria III (Diva software) with $70 \mu \mathrm{m}$ nozzle onto 384 -well capture plates (10\% Triton $\mathrm{X}-100,40 \mathrm{U} / \mu \mathrm{L}$ RNasin plus, dispensed at $4^{\circ} \mathrm{C}$ using the Bravo pipetting robot) with 4 wells that were left empty as control. Since MEP represent more than $90 \%$ of fetal liver LK at this E12.5, the sorting of E12.5 MEP were limited to half of each plate in order to enrich for myeloid progenitors (this selection was not made for sorting of $\mathrm{E} 10.5$ fetal liver). Plates were stored at $-80^{\circ} \mathrm{C}$ until the library preparation step. During library preparation, cDNA from each 384-well plate was divided into two pools termed ' $A$ ' and ' $\mathrm{H}$ ' half-plates which each contained cDNA from 192 uniquely barcoded wells (barcode oligos prepared by Baptiste Saudemont, UTECHS CB). The protocol and reagents for MARS-Seq library preparation have been described in detail (Jaitin et al., 2014; Keren-Shaul et al., 2019).

\section{High-Throughput Sequencing}

DNA quality was tested using an Agilent High Sensitivity D1000 ScreenTape System and quantified using a Qbit 2.0 Fluorometer with dsDNA HS Assay Kit. Libraries were pooled in equimolar amounts (20fmol) per half-plate for a final concentration of $1-2 \mathrm{nM}$. Pooled libraries were sequenced using the NextSeq 500/550 High Output Kit v2 for 75 cycles (Illumina FC-404-2005). Paired-end sequencing was selected with 75 cycles for Read 1 and 16 cycles for Read 2 to capture the barcodes. Base calling accuracy was greater than $80 \%$ ( $>=Q 30,>40 G$ ) and $75-80 \%$ clusters passed filter. Barcode extraction, alignment and generation of QC reports and expression tables was performed by Yann Loe Mie (Bioinformatics and Biostatistics Hub, Institut Pasteur; original pipeline available at http://compgenomics.weizmann.ac.il/tanay/?page_id=672). Data are available on GEO (Series GSE166223).

\section{Cell and Gene Filtering}

The number of UMIs (counts) and features (detected genes) were filtered to eliminate cells with less than 3,000 detected genes. Cells with a mitochondrial gene fraction greater than $5 \%$ were removed. Median Fluorescence Intensity (MFI) values for CD34 and CD16/32 from index sorting were matched to individual well IDs and overlay of cells from each cluster onto two-dimensional flow cytometry plots was performed by defining a bi-exponential scale using ggplot2 (Wickham, 2016). Doublet detection for each plate was performed using default parameters of scDblFinder $R$ package v1.1.8 (https://github.com/plger/scDbIFinder) then filtered to remove cells classified as doublet (Germain et al., 2020). Expressed genes were those detected in at least $5 \%$ of cells from each condition (E10.5_Fetal_Liver, E12.5_Fetal_Liver) were intersected across all plates per condition.

\section{Data Normalization, Merging and Clustering}

Normalization was performed using the deconvolution approach available in the scran package. Cells were pre-clustered within each sequencing run with the 'quickCluster' function using a minimum cluster size of 50 cells and size factors were calculated for each cluster with 'computeSumFactors' of the scran R package v1.15.26 (Lun et al., 2016). The function multiBatchNorm of the batchelor R package v1.3.16 
was then used to rescale the size factors and adjust for differences in sequencing depth between sequencing runs (Lun et al., 2016). The resulting normalized and log-transformed counts were used for further processing. A mean-variance trend was fitted across all genes using 'modelGeneVar' and 'combineVar' functions from the scran $\mathrm{R}$ package with the batches (combination of plate and condition within each sequencing run) used as blocking factor. The top 3,000 genes with the largest positive average biological component across batches were retained. PCA computation was performed on the selected set of genes using 'multiBatchPCA' function of the batchelor package which ensures that each batch contributes equally to the definition of the PC space. Batches were then hierarchically merged using fastMNN to detect mutual nearest neighbors (MNN) of cells in different batches to correct the values in each PCA subspace. Merging was performed using chosen variable genes selected among all expressed genes and detected within at least $5 \%$ of cells across all runs and conditions. Samples were first merged across plates of each condition then 'across conditions' (E10.5 and E12.5). tSNE and UMAP were calculated with Seurat v3.2.2 on fastMNN reduction (Stuart et al., 2018). Clustering was obtained by random walks (function 'cluster_walktrap' of the igraph v1.2.5 package) (Pons and Latapy, 2005) on a shared nearest-neighbor (SNN) graph (built with 'buildSNNGraph' function of the scran package using $\mathrm{k}=12$ nearest neighbors). Markers for each cluster were identified using pairwise t-tests to keep genes that were differentially expressed in at least half of the pairwise comparisons with the other clusters ('findMarkers' function of the scran package with option 'pval.type="some"'). The overlap between markers across clusters was studied using the R package UpSetR v1.4.0 (Conway et al., 2017). Correlation of the cluster average expression profiles with those of the Mouse Cell Atlas was made with the reference matrix provided in the ScMCA v0.2.0 package (https://github.com/ggjlab/scMCA) and exclusion of cell types that matched less than 10 cells.

\section{Integration of datasets}

For integrations with external datasets, cells were loaded from GSE125088 (Yahara et al., 2020) and GSE81760 (Mass et al., 2016). Sample information was extracted from the barcode cell ID and cells were additionally filtered to exclude those with $>5 \%$ mitochondrial genes and/or $<2,000$ detected genes. Only the Csf1rmericreMer Rosa26 tdTomato Csf1r EGFP genotypes were kept. Doublets were removed as described earlier and data was normalized by library-size normalization. Cluster and cell type information were unavailable, therefore we assigned tissue and cell type based on comparative annotation using the scMCA v0.2.0 package. Only cells annotated as fetal liver or peripheral blood myeloid cells were kept for integration. Data from GSE81760 were already filtered and normalized. To account for different versions of the MGI databases, we input MGI symbols for all genes from each dataset onto the Mouse Genome Database (MGD) website of the Jackson Laboratory (http://www.informatics.jax.org/batch, May 2020; Bult et al., 2019) to get their matching gene ensembl id then intersected the gene labels of the three datasets. We built a common dataset based on common genes and applied the function 'multiBatchNorm ' to adjust for differences in sequencing depth between plates. Datasets were then integrated using fastMNN using the intersection with the genes selected as highly variable in our dataset. Samples were merged by dataset. 


\section{Single Cell CFU Assays}

Cells were isolated and prepared for flow cytometry as described above. Red blood cell lysis was performed for E18.5. Lineage cells (Ter119+ $\mathrm{CD} 19^{+} \mathrm{CD} 3 \mathrm{e}^{+} \mathrm{NK} 1.1^{+} \mathrm{F} 4 / 80^{+} \mathrm{Gr} 1^{+}$) were depleted using magnetic anti-biotin Microbeads (1:5) (Miltenyi Biotec 130-090-485) and MS columns (Miltenyi Biotec 130-042-201). Cells were sorted using a FACSAria III (Diva software) and cloned into flat-bottom 96well plates containing pre-warmed and equilibrated differentiation medium $(0.1 \% \beta$-mercaptoethanol,

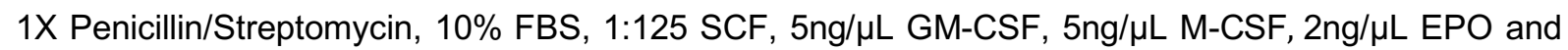
$5 \mathrm{ng} / \mu \mathrm{L}$ TPO in Opti-MEM with Glutamax). SCF was supplied from myeloma cell line supernatant. Cells were grown at $37^{\circ} \mathrm{C}$ with $5 \% \mathrm{CO}_{2}$ for 7 days. Wells were manually scored for the presence of colonies and cells were collected by scratching for flow cytometry analysis.

\section{Serial Dilution CFU Assays}

Single cell CFU assays were performed as described above except that cells were supplemented with fresh differentiation media on day 6 and analyzed on day 8 after sorting. Growth factors and cytokines were used at the same concentration with the exception of SCF that was further diluted in a series of 1:8 and 1:64. Each 96-well plate used for clonal sorting contained 4 columns (32 wells) per condition $(1 \mathrm{x}, 1: 8,1: 64 \mathrm{SCF})$ to minimize plate effects.

\section{Adoptive Transfer}

Myeloid progenitors $\left(\mathrm{Lin}^{\text {neg }} \mathrm{Sca} 1^{\text {neg }} \mathrm{Kit}^{+} \mathrm{CD} 34^{+} \mathrm{CD} 16 / 32^{+}\right)$were isolated and prepared for flow cytometry as described above. Red blood cell lysis was performed for E18.5. Lineage cells (Ter119+ $\mathrm{CD} 19^{+} \mathrm{CD} 3 \mathrm{e}^{+} \mathrm{NK} 1.1^{+} \mathrm{F} 4 / 80^{+} \mathrm{Gr} 1^{+}$) were depleted using magnetic anti-biotin Microbeads (1:5) (Miltenyi Biotec 130-090-485) and MS columns (Miltenyi Biotec 130-042-201). Cells were sorted in 0.1\% FBS using a FACSAria III (Diva software) from E11.5 Rosa26 ${ }^{m T m G}$ CD45.2/2 donor embryos. Neonatal (P0) Rag2 2-; $\mathrm{YC}^{-/-}$CD45.1/1 pups were irradiated (2 Grey; Xstrahl X-ray system) 2 hours prior to injection. Approximately 20,000 cells were transferred intravenously to the facial vein using a Hamilton syringe. Organs were isolated 5 days later and analyzed by flow cytometry as described above.

\section{Data Availability Statement}

Original scRNAseq data reported in this study is deposited in the NCBI Geo under ID code: GSE166223 (https://www.ncbi.nlm.nih.gov/geo/query/acc.cgi?acc=GSE166223). scRNAseq data from external datasets (Fig. 3-4 and Extended data Fig.3-4) are publicly available (GSE125088, GSE81760, GSE72857). All other source data is available upon request.

\section{Code Availability Statement}

All code used for scRNAseq analysis will be made available to the public at the time of publication at https://www.ncbi.nlm.nih.gov/geo/query/acc.cgi?acc=GSE166223.

\section{References}

Ajami, B., Bennett, J.L., Krieger, C., McNagny, K.M., and Rossi, F.M.V. (2011). Infiltrating monocytes trigger EAE progression, but do not contribute to the resident microglia pool. Nat. Neurosci. 14, 1142-1150. 
Akashi, K., Traver, D., Miyamoto, T., and Weissman, I.L. (2000). A clonogenic common myeloid progenitor that gives rise to all myeloid lineages. Nature 404, 193-197.

Ana Cumano, Francoise Dieterian-Lievre, and Isabelle Godin (1996). Lymphoid Potential, Probed before Circulation in Mouse, Is Restricted to Caudal Intraembryonic Splanchnopleura. Cell 86, 907-916.

Andrade, E.B., Alves, J., Madureira, P., Oliveira, L., Ribeiro, A., Cordeiro-da-Silva, A., Correia-Neves, M., Trieu-Cuot, P., and Ferreira, P. (2013). TLR2-Induced IL-10 Production Impairs Neutrophil Recruitment to Infected Tissues during Neonatal Bacterial Sepsis. J. Immunol. 191, 4759-4768.

Azzoni, E., Frontera, V., McGrath, K.E., Harman, J., Carrelha, J., Nerlov, C., Palis, J., Jacobsen, S.E.W., and Bruijn, M.F. (2018). Kit ligand has a critical role in mouse yolk sac and aorta-gonad-mesonephros hematopoiesis. EMBO Rep. 19, 1-17. Beaudin, A.E., Boyer, S.W., Perez-Cunningham, J., Hernandez, G.E., Derderian, S.C., Jujjavarapu, C., Aaserude, E., MacKenzie, T., and Forsberg, E.C. (2016). A Transient Developmental Hematopoietic Stem Cell Gives Rise to Innate-like B and T Cells. Cell Stem Cell 19, 768-783

Bennett, F.C., Bennett, M.L., Yaqoob, F., Mulinyawe, S.B., Grant, G.A., Hayden Gephart, M., Plowey, E.D., and Barres, B.A. (2018). A Combination of Ontogeny and CNS Environment Establishes Microglial Identity. Neuron 98, 1170-1183.e8.

De Bruijn, M.F.T.R., Speck, N.A., Peeters, M.C.E., and Dzierzak, E. (2000). Definitive hematopoietic stem cells first develop within the major arterial regions of the mouse embryo. EMBO J. 19, 2465-2474.

Bult, C.J., Blake, J.A., Smith, C.L., Kadin, J.A., Richardson, J.E., Anagnostopoulos, A., Asabor, R., Baldarelli, R.M., Beal, J.S., Bello, S.M., et al. (2019). Mouse Genome Database (MGD) 2019. Nucleic Acids Res. 47, D801-D806.

Chen, M.J., Li, Y., De Obaldia, M.E., Yang, Q., Yzaguirre, A.D., Yamada-Inagawa, T., Vink, C.S., Bhandoola, A., Dzierzak, E. and Speck, N.A. (2011). Erythroid/myeloid progenitors and hematopoietic stem cells originate from distinct populations of endothelial cells. Cell Stem Cell 9, 541-552.

Conway, J.R., Lex, A., and Gehlenborg, N. (2017). UpSetR: An R package for the visualization of intersecting sets and their properties. Bioinformatics 33, 2938-2940.

Cumano, A., Ferraz, J.C., Klaine, M., Di Santo, J.P., and Godin, I. (2001). Intraembryonic, but not yolk sac hematopoietic precursors, isolated before circulation, provide long-term multilineage reconstitution. Immunity 15, 477-485.

Dahlin, J.S., Hamey, F.K., Pijuan-Sala, B., Shepherd, M., Lau, W.W.Y., Nestorowa, S., Weinreb, C., Wolock, S., Hannah, R. Diamanti, E., et al. (2018). A single-cell hematopoietic landscape resolves 8 lineage trajectories and defects in Kit mutant mice. Blood 131, e1-e11.

Dege, C., Fegan, K.H., Creamer, J.P., Berrien-Elliott, M.M., Luff, S.A., Kim, D., Wagner, J.A., Kingsley, P.D., McGrath, K.E., Fehniger, T.A., et al. (2020). Potently Cytotoxic Natural Killer Cells Initially Emerge from Erythro-Myeloid Progenitors during Mammalian Development. Dev. Cell 53, 229-239.e7.

Elsaid, R., Soares-Da-Silva, F., Peixoto, M., Amiri, D., Mackowski, N., Pereira, P., Bandeira, A., and Cumano, An. (2020). Hematopoiesis: a layered organization across species. Front. Cell Dev. Biol. 8, 1557.

Elsaid, R., Meunier, S., Burlen-defranoux, O., Perchet, T., Iturri, L., Freyer, L., Vieira, P., Pereira, P., Golub, R., Bandeira, A., et al. (2021). A wave of bipotent T / ILC-restricted progenitors shapes the embryonic thymus microenvironment in a timedependent manner A wave of bipotent T / ILC-restricted progenitors shapes the embryonic thymus microenvironment in a timedependent manner. Blood.

Frame, J.M. (2015). Definitive Hematopoiesis in the Yolk Sac Emerges from Wnt-Responsive Hemogenic Endothelium Independently of Circulation and Arterial Identity.

Gao, X., Xu, C., Asada, N., and Frenette, P.S. (2018). The hematopoietic stem cell niche: From embryo to adult. Dev. 145. Gekas, C., Dieterlen-Lièvre, F., Orkin, S.H., and Mikkola, H.K.A. (2005). The placenta is a niche for hematopoietic stem cells. Dev. Cell 8, 365-375

Gentek, R., Ghigo, C., Hoeffel, G., Bulle, M.J., Msallam, R., Gautier, G., Launay, P., Chen, J., Ginhoux, F., and Bajénoff, M. (2018). Hemogenic Endothelial Fate Mapping Reveals Dual Developmental Origin of Mast Cells. Immunity 48, 1160-1171.e5. Germain, P.L., Sonrel, A., and Robinson, M.D. (2020). PipeComp, a general framework for the evaluation of computational pipelines, reveals performant single-cell RNA-seq preprocessing tools. BioRxiv.

Ginhoux, F., Greter, M., Leboeuf, M., Nandi, S., See, P., Gokhan, S., Mehler, M.F., Conway, S.J., Ng, L.G., Stanley, E.R., et al. (2010). Primitive Macrophages. Science (80-. ). 701, 841-845.

Gomez Perdiguero, E., Klapproth, K., Schulz, C., Busch, K., Azzoni, E., Crozet, L., Garner, H., Trouillet, C., De Bruijn, M.F., Geissmann, F., et al. (2015). Tissue-resident macrophages originate from yolk-sac-derived erythro-myeloid progenitors. Nature 518, 547-551.

Hamey, F.K., Lau, W.W.Y., Kucinski, I., Wang, X., Diamanti, E., Wilson, N.K., Göttgens, B., and Dahlin, J.S. (2020). Single-cell molecular profiling provides a high-resolution map of basophil and mast cell development. Allergy Eur. J. Allergy Clin. Immunol. $1-12$.

Han, X., Wang, R., Zhou, Y., Fei, L., Sun, H., Lai, S., Saadatpour, A., Zhou, Z., Chen, H., Ye, F., et al. (2018). Mapping the Mouse Cell Atlas by Microwell-Seq. Cell 172, 1091-1107.e17.

Hashimoto, D., Chow, A., Noizat, C., Teo, P., Beasley, M.B., Leboeuf, M., Becker, C.D., See, P., Price, J., Lucas, D., et al. (2013). Tissue-resident macrophages self-maintain locally throughout adult life with minimal contribution from circulating monocytes. Immunity 38, 792-804.

Hoeffel, G., Chen, J., Lavin, Y., Low, D., Almeida, F.F., See, P., Beaudin, A.E., Lum, J., Low, I., Forsberg, E.C., et al. (2015). CMyb+ Erythro-Myeloid Progenitor-Derived Fetal Monocytes Give Rise to Adult Tissue-Resident Macrophages. Immunity 42, 665-678.

Honold, L., and Nahrendorf, M. (2018). Resident and Monocyte-Derived Macrophages in Cardiovascular Disease. Circ. Res. 122, 113-127.

Iturri, L., Freyer, L., Biton, A., Dardenne, P., Lallemand, Y., and Gomez Perdiguero, E. (2021). Two sequential and independent pathways of erythromyeloid Summary : Immunity.

Jaitin, D.A., Kenigsberg, E., Keren-Shaul, H., Elefant, N., Paul, F., Zaretsky, I., Mildner, A., Cohen, N., Jung, S., Tanay, A., et al. (2014). Massively parallel single-cell RNA-seq for marker-free decomposition of tissues into cell types. Science (80-. ). 343, $776-779$.

Kasaai, B., Caolo, V., Peacock, H.M., Lehoux, S., Gomez-Perdiguero, E., Luttun, A., and Jones, E.A.V. (2017). Erythro-myeloid progenitors can differentiate from endothelial cells and modulate embryonic vascular remodeling. Sci. Rep. 7, 25-28.

Keren-Shaul, H., Kenigsberg, E., Jaitin, D.A., David, E., Paul, F., Tanay, A., and Amit, I. (2019). MARS-seq2.0: an experimental and analytical pipeline for indexed sorting combined with single-cell RNA sequencing. Nat. Protoc. 14, 1841-1862.

Kieusseian, A., de la Grange, P.B., Burlen-Defranoux, O., Godin, I., and Cumano, A. (2012). Immature hematopoietic stem cells undergo maturation in the fetal liver. Dev. 139, 3521-3530.

Krow-Lucal, E.R., Kim, C.C., Burt, T.D., and McCune, J.M. (2014). Distinct functional programming of human fetal and adult 
monocytes. Blood 123, 1897-1904.

Laughlin, M.J., Barker, J., Bambach, B., Koc, O.N., Rizzieri, D.A., Wagner, J.E., Gerson, S.L., Lazarus, H.M., Cairo, M., Stevens, C.E., et al. (2001). Hematopoietic Engraftment and Survival in Adult Recipients of Umbilical-Cord Blood From Unrelated Donors. Obstet. Gynecol. Surv. 56, 687-688.

Levy, O. (2007). Innate immunity of the newborn: Basic mechanisms and clinical correlates. Nat. Rev. Immunol. 7, 379-390.

Li, Z., Liu, S., Xu, J., Zhang, X., Han, D., Liu, J., Xia, M., Yi, L., Shen, Q., Xu, S., et al. (2018). Adult Connective Tissue-Resident Mast Cells Originate from Late Erythro-Myeloid Progenitors. Immunity 49, 640-653.e5.

Loyher, P.L., Hamon, P., Laviron, M., Meghraoui-Kheddar, A., Goncalves, E., Deng, Z., Torstensson, S., Bercovici, N., De

Chanville, C.B., Combadière, B., et al. (2018). Macrophages of distinct origins contribute to tumor development in the lung. J.

Exp. Med. 215, 1-18.

Lun, A.T.L., McCarthy, D.J., and Marioni, J.C. (2016). A step-by-step workflow for low-level analysis of single-cell RNA-seq data [version 1; referees: 5 approved with reservations]. F1000Research 5.

Lux, C.T., Yoshimoto, M., McGrath, K., Conway, S.J., Palis, J., and Yoder, M.C. (2008). All primitive and definitive

hematopoietic progenitor cells emerging before E10 in the mouse embryo are products of the yolk sac. Blood 111, 3435-3438.

Mass, E., and Gentek, R. (2021). Fetal-Derived Immune Cells at the Roots of Lifelong Pathophysiology. Front. Cell Dev. Biol. 9, $1-17$.

Mass, E., Ballesteros, I., Farlik, M., Halbritter, F., Günther, P., Crozet, L., Jacome-Galarza, C.E., Händler, K., Klughammer, J., Kobayashi, Y., et al. (2016). Specification of tissue-resident macrophages during organogenesis. Science (80-. ). 353.

McGrath, K.E., Fegan, K., Catherman, S., and Palis, J. (2014). Emergence of the neutrophil lineage in the mammalian embryo. Exp. Hematol. 42, S13.

McGrath, K.E., Frame, J.M., Fegan, K.H., Bowen, J.R., Conway, S.J., Catherman, S.C., Kingsley, P.D., Koniski, A.D., and Palis, J. (2015). Distinct Sources of Hematopoietic Progenitors Emerge before HSCs and Provide Functional Blood Cells in the Mammalian Embryo. Cell Rep. 11, 1892-1904.

McKercher, S.R., Torbett, B.E., Anderson, K.L., Henkel, G.W., Vestal, D.J., Baribault, H., Klemsz, M., Feeney, A.J., Wu, G.E., Paige, C.J., et al. (1996). Targeted disruption of the PU.1 gene results in multiple hematopoietic abnormalities. EMBO J. 15, $5647-5658$

Medvinsky, A., and Dzierzak, E. (1996). Definitive hematopoiesis is autonomously initiated by the AGM region. Cell 86, 897906.

Msallam, R., Balla, J., Rathore, A.P.S., Kared, H., Malleret, B., Saron, W.A.A., Liu, Z., Hang, J.W., Dutertre, C.A., Larbi, A., et al. (2020). Fetal mast cells mediate postnatal allergic responses dependent on maternal IgE. Science (80-. ). 370, 941-950. Ogawa, M., Nishikawa, S., Yoshinaga, K., Hayashi, S.I., Kunisada, T., Nakao, J., Kina, T., Sudo, T., Kodama, H., and Nishikawa, S.I. (1993). Expression and function of c-Kit in fetal hemopoietic progenitor cells: Transition from the early c-Kitindependent to the late c-Kit-dependent wave of hemopoiesis in the murine embryo. Development 117, 1089-1098.

Ottersbach, K., and Dzierzak, E. (2005). The murine placenta contains hematopoietic stem cells within the vascular labyrinth region. Dev. Cell 8, 377-387.

Palis, J., Robertson, S., Kennedy, M., Wall, C., and Keller, G. (1999). Development of erythroid and myeloid progenitors in the yolk sac and embryo proper of the mouse. Development 126, 5073-5084.

Paul, F., Arkin, Y., Giladi, A., Jaitin, D.A., Kenigsberg, E., Keren-Shaul, H., Winter, D., Lara-Astiaso, D., Gury, M., Weiner, A., et al. (2015). Transcriptional Heterogeneity and Lineage Commitment in Myeloid Progenitors. Cell 163, 1663-1677.

Perdiguero, E.G., and Geissmann, F. (2016). The development and maintenance of resident macrophages. Nat. Immunol. 17, 2-8.

Pons, P., and Latapy, M. (2005). Computing communities in large networks using random walks. Lect. Notes Comput. Sci. (Including Subser. Lect. Notes Artif. Intell. Lect. Notes Bioinformatics) 3733 LNCS, 284-293.

Schulz, C., Perdiguero, E.G., Chorro, L., Szabo-Rogers, H., Cagnard, N., Kierdorf, K., Prinz, M., Wu, B., Jacobsen, S.E.W., Pollard, J.W., et al. (2012). A lineage of myeloid cells independent of myb and hematopoietic stem cells. Science (80-. ). 335, 86-90.

Simic, M., Manosalva, I., Spinelli, L., Gentek, R., Shayan, R.R., Siret, C., Girard-Madoux, M., Wang, S., de Fabritus, L., Verschoor, J., et al. (2020). Distinct Waves from the Hemogenic Endothelium Give Rise to Layered Lymphoid Tissue Inducer Cell Ontogeny. Cell Rep. 32, 108004.

Soares-Da-Silva, F., Freyer, L., Elsaid, R., Burlen-Defranoux, O., Iturri, L., Sismeiro, O., Pinto-Do, P., Gomez-Perdiguero, E., and Cumano, A. (2021). Yolk sac, but not hematopoietic stem cell-derived progenitors, sustain erythropoiesis throughout murine embryonic life. J. Exp. Med. 218.

Stremmel, C., Schuchert, R., Wagner, F., Thaler, R., Weinberger, T., Pick, R., Mass, E., Ishikawa-Ankerhold, H.C., Margraf, A., Hutter, S., et al. (2018). Yolk sac macrophage progenitors traffic to the embryo during defined stages of development. Nat. Commun. 9.

Stuart, T., Butler, A., Hoffman, P., Hafemeister, C., Papalexi, E., Mauck, W.M., Stoeckius, M., Smibert, P., and Satija, R. (2018) Comprehensive integration of single cell data. BioRxiv 1-24.

Traver, D., Miyamoto, T., Christensen, J., Iwasaki-Arai, J., Akashi, K., and Weissman, I.L. (2001). Fetal liver myelopoiesis occurs through distinct, prospectively isolatable progenitor subsets. Blood 98, 627-635.

Wickham, H. (2016). ggplot2: Elegant Graphics for Data Analysis (Springer-Verlag New York).

Yahara, Y., Barrientos, T., Tang, Y.J., Puviindran, V., Nadesan, P., Zhang, H., Gibson, J.R., Gregory, S.G., Diao, Y., Xiang, Y., et al. (2020). Erythromyeloid progenitors give rise to a population of osteoclasts that contribute to bone homeostasis and repair. Nat. Cell Biol. 22, 49-59.

Yáñez, A., Coetzee, S.G., Olsson, A., Muench, D.E., Berman, B.P., Hazelett, D.J., Salomonis, N., Grimes, H.L., and Goodridge, H.S. (2017). Granulocyte-Monocyte Progenitors and Monocyte-Dendritic Cell Progenitors Independently Produce Functionally Distinct Monocytes. Immunity 47, 890-902.e4.

Yona, S., Kim, K.W., Wolf, Y., Mildner, A., Varol, D., Breker, M., Strauss-Ayali, D., Viukov, S., Guilliams, M., Misharin, A., et al. (2013). Fate Mapping Reveals Origins and Dynamics of Monocytes and Tissue Macrophages under Homeostasis. Immunity 38 , 79-91.

Yvernogeau, L., and Robin, C. (2017). Restricted intra-embryonic origin of bona fide hematopoietic stem cells in the chicken. Dev. 144, 2352-2363.

Zhu, Y., Herndon, J.M., Sojka, D.K., Kim, K.W., Knolhoff, B.L., Zuo, C., Cullinan, D.R., Luo, J., Bearden, A.R., Lavine, K.J., et al. (2017). Tissue-Resident Macrophages in Pancreatic Ductal Adenocarcinoma Originate from Embryonic Hematopoiesis and Promote Tumor Progression. Immunity 47, 323-338.e6. 
Fig. 1

a.
EMP-derived Mature Cells
HSC-derived Mature Cells
Cdh5 ${ }^{\text {CreERT2 }}$ Rosa26 $6^{\text {YFP }}$
FETAL LIVER

All Cells

Neutrophils
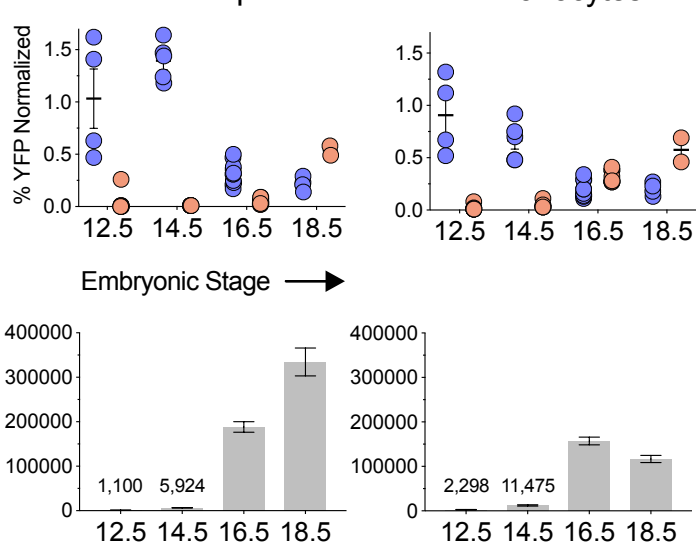

c.
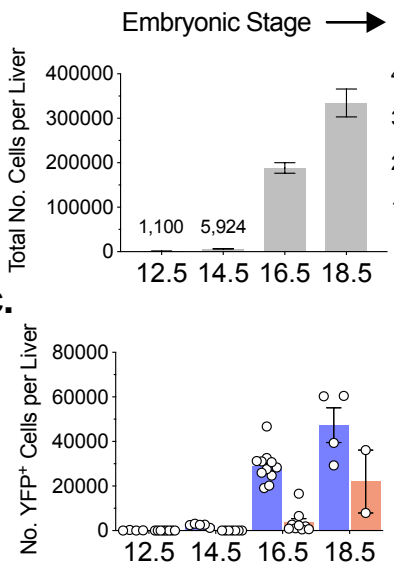

d.
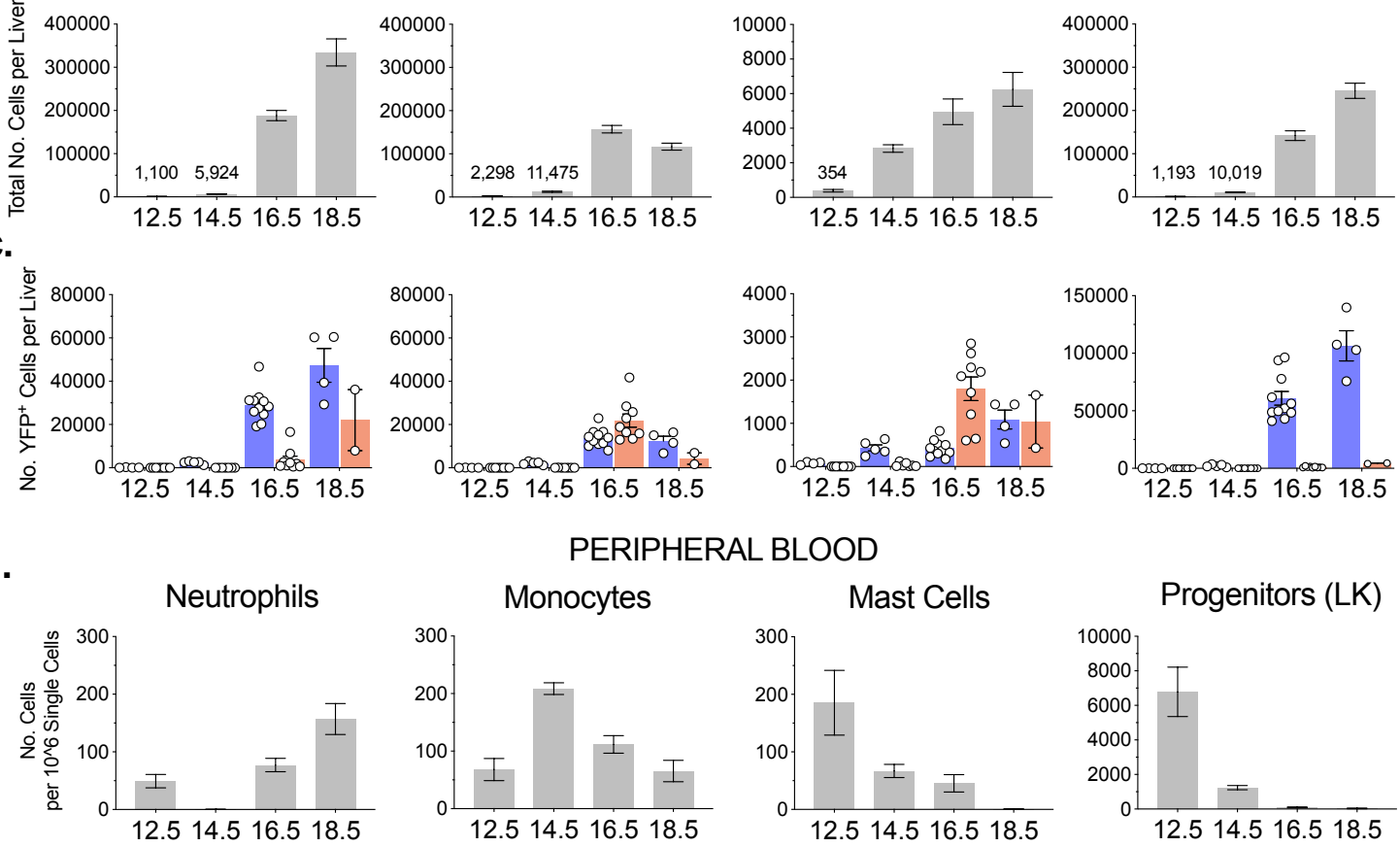

PERIPHERAL BLOOD

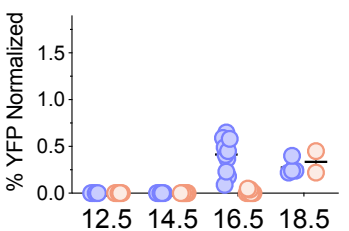

Monocytes
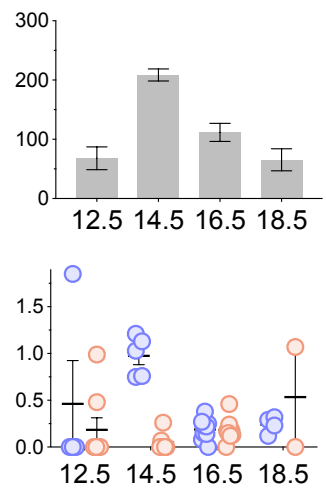

Mast Cells
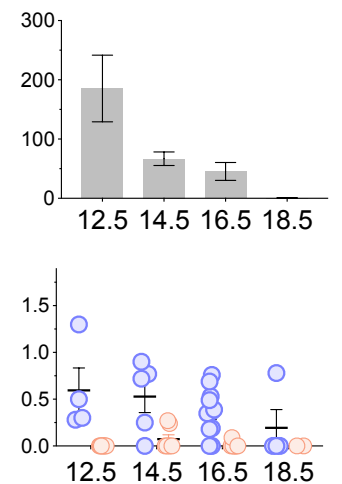

Fig. 1. The EMP-to-HSC switch in myelopoiesis occurs late and is lineage specific. a-e, In vivo pulse chase labeling using Cdh5 CreERT2 ROSa26 YFP with E7.5 OHT injection (to label EMP-derived cells) or E10.5 OHT injection (to label HSC-derived cells). YFP labeling among mature myeloid cells normalized to YFP labeling of microglia (for E7.5 OHT) or normalized to YFP labeling of LSK (for E10.5 OHT) (a). Total number of mature myeloid cells (YFP labeled and unlabeled) per fetal liver (b). Number of YFP+ mature myeloid cells per fetal liver (c). Relative number of mature myeloid cells and LK pan-progenitors (YFP labeled and unlabeled) per $1 \times 10^{\wedge} 6$ single cells in peripheral blood circulation (d). YFP labeling of mature myeloid cells in peripheral blood circulation as normalized in panel a (e). For E7.5 OHT injections, E12.5 $n=4$; E14.5 $n=5$; E16.5 $n=11$; E18.5 $n=4$. For E10.5 OHT injections, E12.5 $n=8$; E14.5 $n=7$; E16.5 $n=9$; E18.5 $n=2$. $n$ represents biologically independent embryos from 1-2 independent experiments. Data are represented as mean \pm s.e.m. Mean values are written above some bars. 
Fig. 2

a.

E14.5 FETAL LIVER Lin $^{\text {neg }}$ Sca1 ${ }^{\text {neg }} \mathrm{Kit}^{+}(\mathbf{L K})$

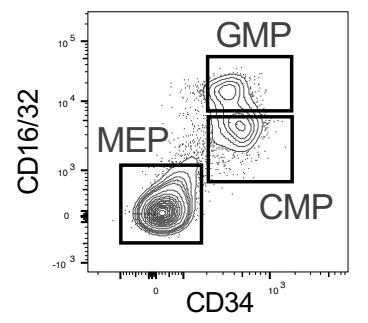

b.

Lin $^{\text {neg }}$ Sca1 ${ }^{\text {neg }} \mathrm{Kit}^{+}$ (LK)

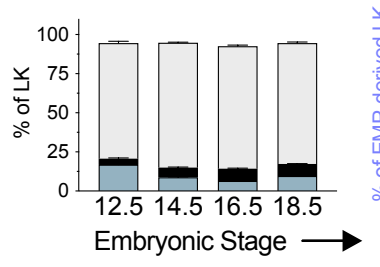

c.

EMP-derived Progenitors HSC-derived Progenitors

CMP
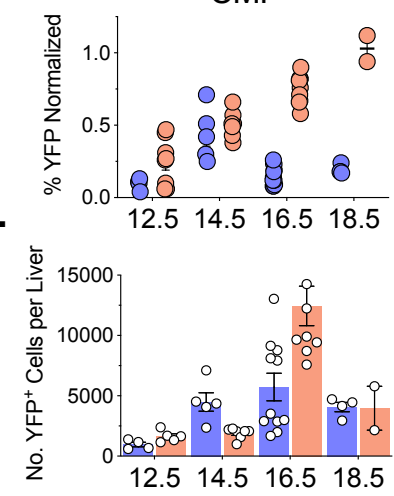

e.

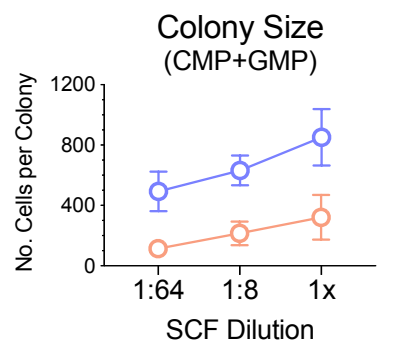

EMP-derived LK (E7.5 OHT YFP+ LK)

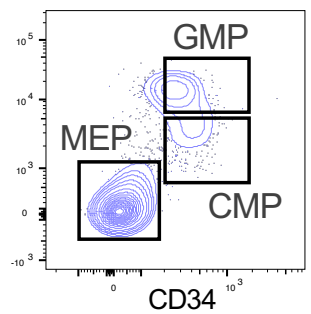

EMP-derived LK (E7.5 OHT YFP+ LK)

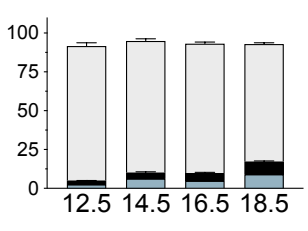

Cdh5 ${ }^{\text {CreERT2 Rosa26 YFP }}$

FETAL LIVER

GMP
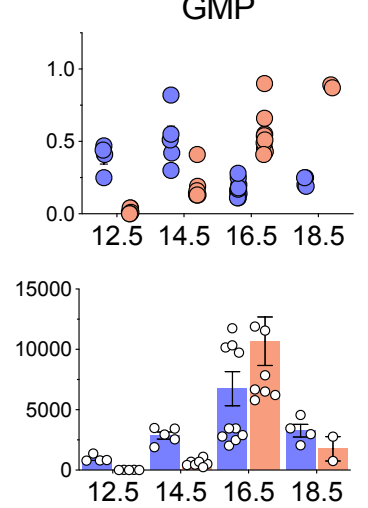

f.

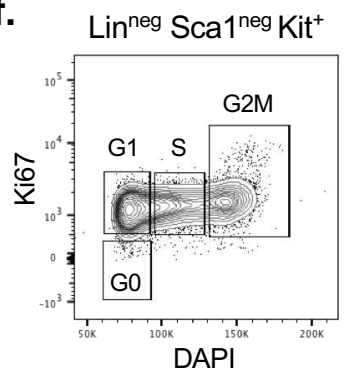

HSC-derived LK (E10.5 OHT YFP+ LK)

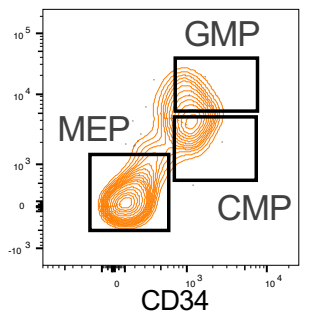

HSC-derived LK (E10.5 OHT YFP+ LK)

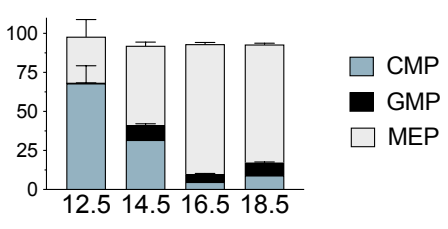

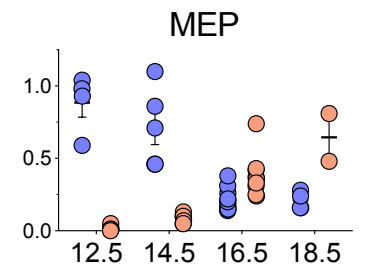

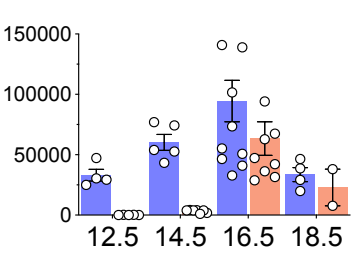

E11.5

E11.5

FETAL LIVER PERIPHERAL

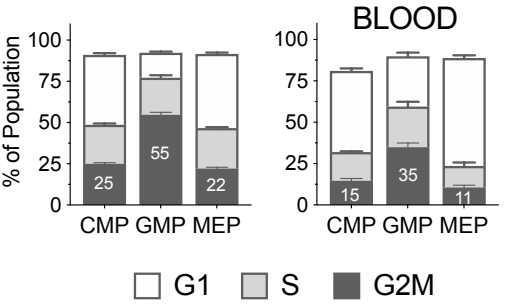

Fig. 2. Expansion of EMP progeny drives myeloid progenitor diversity in the early fetal liver. a-d, In vivo pulse chase labeling using Cdh5CreERT2 ROsa26 YFP with E7.5 OHT injection (to label EMP-derived cells) or E10.5 OHT injection (to label HSC-derived cells). Immunophenotyping of CMP, GMP and MEP in the fetal liver with overlay of pulse labeled $\mathrm{YFP}^{+}$cells (a). Proportions of total and pulse labeled CMP, GMP and MEP (b). YFP labeling (normalized as in Fig. 1) of CMP, GMP and MEP (c). Number of YFP+ progenitors per fetal liver (d). Numbers of biological samples are the same as in Fig. 1. e, Single-cell CFU assay with myeloid progenitors sorted from E11.5 (EMP-derived) and E18.5 (HSC-derived) fetal liver. Colony size with saturating $(1 \mathrm{x}$; E11.5 $n=115$, E18.5 $n=90)$ and diluted concentrations of SCF (1:8; $\mathrm{E} 11.5 n=98, \mathrm{E} 18.5 n=84$ and 1:64; E11.5 $n=81, \mathrm{E} 18.5 n=87 . n$ represents numbers of analyzed colonies from one independent experiment per stage. $\mathbf{f}$, Cell cycle analysis of the E11.5 fetal liver and peripheral blood using Ki67 with DAPI staining. E11.5 $n=9$ biologically independent animals from one experiment. Data are represented as mean \pm s.e.m. Mean values are written over some bars. 
Fig. 3

a.

EMP-derived (E8.5 OHT) EMP-derived (E9.5 OHT) CMP

b.
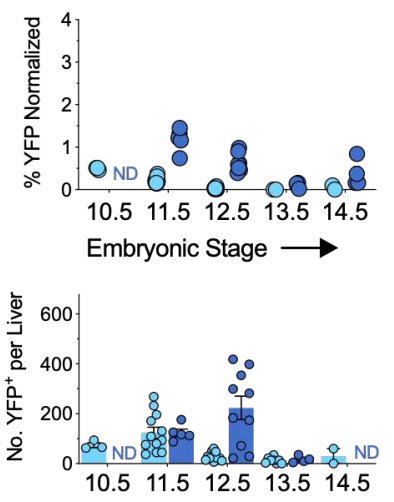

c.

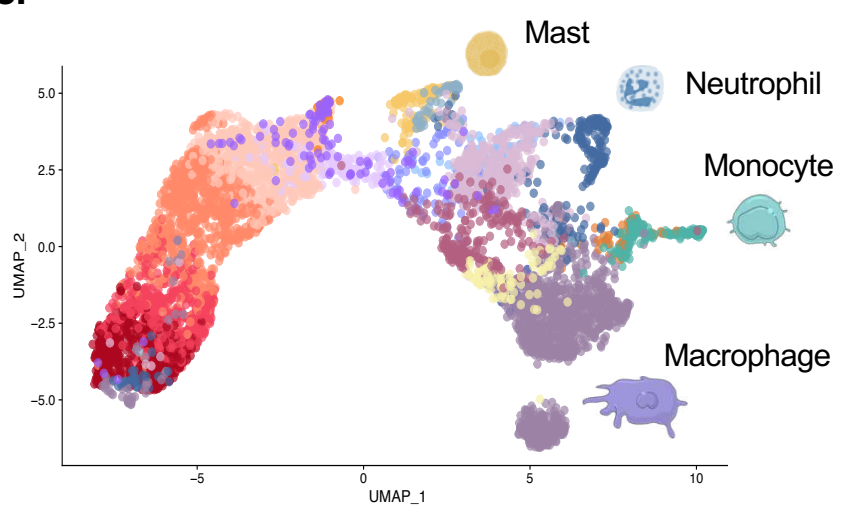

Csf1r MeriCreMer Rosa26 YFP

FETAL LIVER

GMP
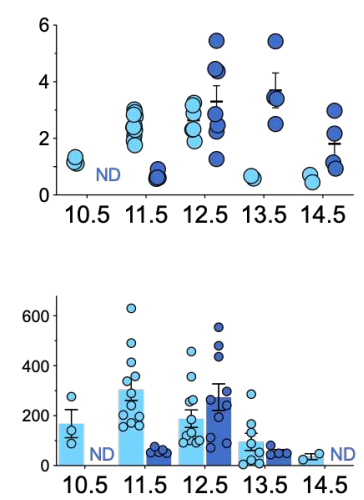

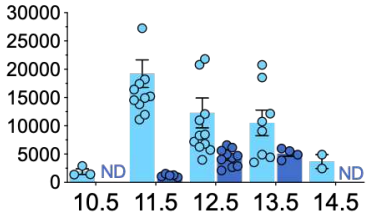

Freyer et al., 2021 Mass et al., 2016

1 EMP
2 MP
3 GP
4 Mac
5 Mast
6 EP
7 early_Ery
8 mid_Ery
9
10 late_Ery
10 MkP

11 EMP

$12 \mathrm{pMac}$

$13 \mathrm{Mac}$

Yahara et al., 2020

14 Mast (Mcpt8 $\left.{ }^{\text {hi }}\right)$

$15 \mathrm{Nt}$ (Elane $\left.{ }^{\text {hi}}\right)$

$16 \mathrm{Nt}\left(\mathrm{Ngp}^{\text {hi }}\right)$

$17 \mathrm{MF}$

18 MF (Flt-ps)

19 MF/MO (S100a4 $\left.{ }^{\text {hi }}\right)$

d.

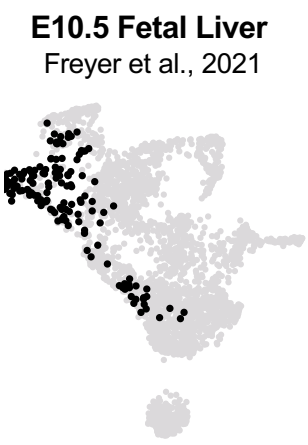

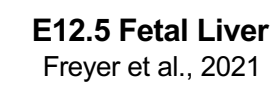

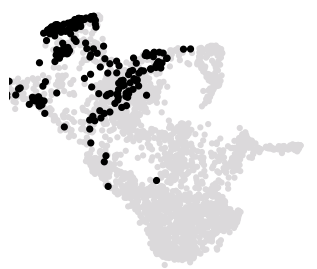

E10.25 Embryo Mass et al., 2016

\section{E14.5 Fetal Liver}

Yahara et al., 2020

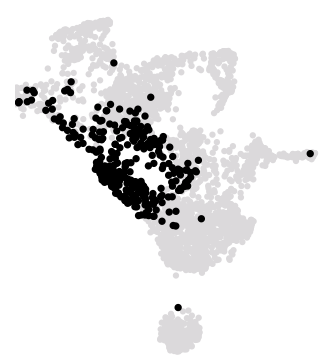

Fig. 3. Single cell transcriptomic analysis of EMP-derived cells in the fetal liver. a-b, In vivo pulse chase labeling using Csf1rmericremer Rosa26 ${ }^{\text {YFP }}$ with E8.5 OHT or E9.5 OHT injection. Percentage of YFP labeling of CMP, MEP and GMP normalized to labeling of EMP-derived megakaryocytes (a) and number of YFP ${ }^{+}$cells per fetal liver (b). ND, no data. For E8.5 OHT injections, E10.5 $n=4$; E11.5 $n=12$; E12.5 $n$ $=12 ; \mathrm{E} 13.5 n=8 ; \mathrm{E} 14.5 n=2$. For E9.5 OHT injections, E11.5 $n=5$; E12.5 $n=10 ; \mathrm{E} 13.5 n=4 ; \mathrm{E} 14.5 n$ $=4$. $n$ represents biologically independent embryos from 1-2 independent experiments. Data are represented as mean \pm s.e.m. c-d, UMAP representation of scRNAseq analysis of pulse labeled LK isolated from Csf1rmericremer Rosa26 YFP embryos injected with E8.5 OHT (1,590 cells from E10.5 fetal liver and 1,405 cells from E12.5 fetal liver). Cells from our dataset (clusters 1-10) were integrated with external datasets including E10.25 CD45 cells (Mass et al., clusters 11-13) and E14.5 pulse labeled fetal liver myeloid cells (Yahara et al., clusters 14-19) (c). Individual datatsets and embryonic stages are highlighted (black) to show progression from progenitors to mature myeloid states (d). EMP, erythromyeloid progenitor; MP, myeloid progenitor; GP, granulocyte progenitor; Mac, macrophage; EP, erythroid progenitor; Ery, erythroid; MkP, megakaryocyte progenitor; pMac, pre-macrophage; Nt, neutrophil; MF, macrophage; MO, monocyte. 
Fig. 4

a.

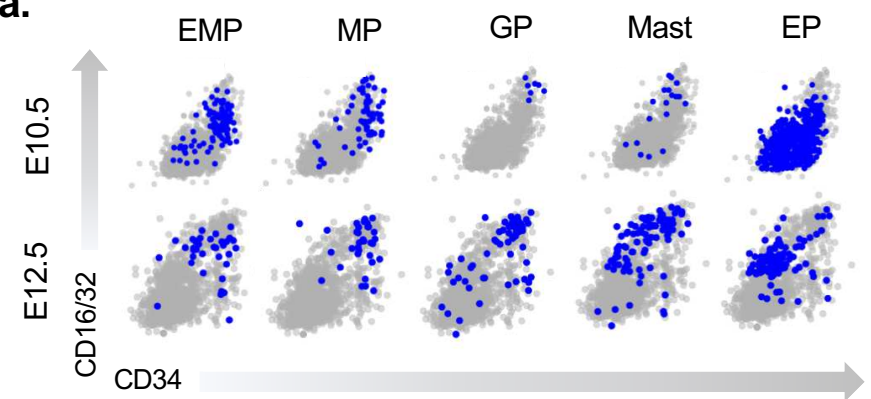

b.

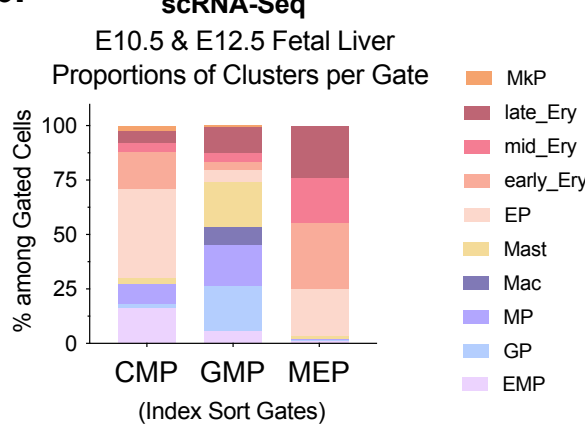

c.

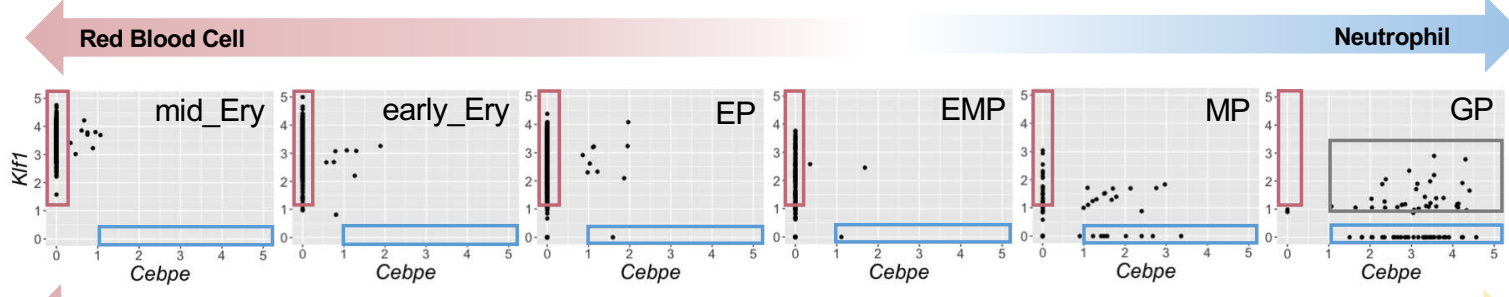

Red Blood Cell

Mast Cell

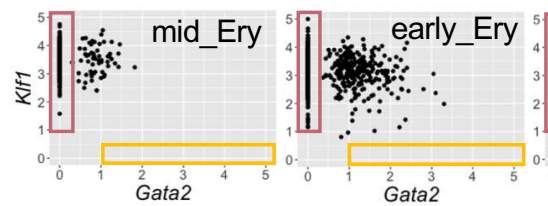

d.

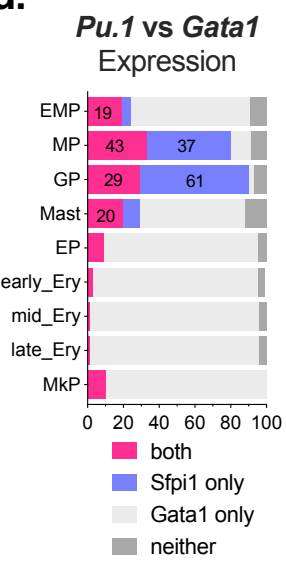

KIf1 vs Cebpe Expression

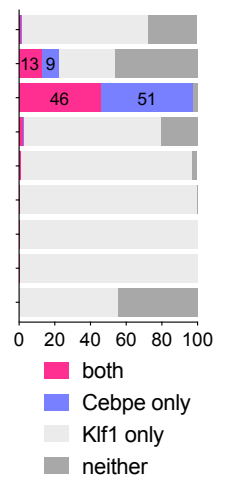

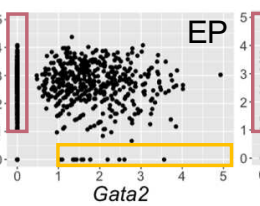

KIf1 vs Gata2 Expression

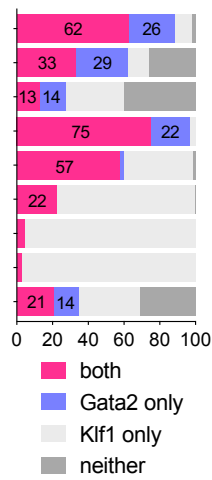

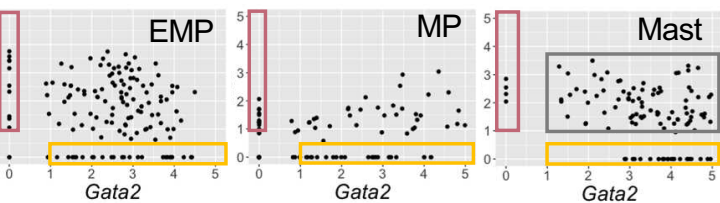

e.

Clonal CFU Assay

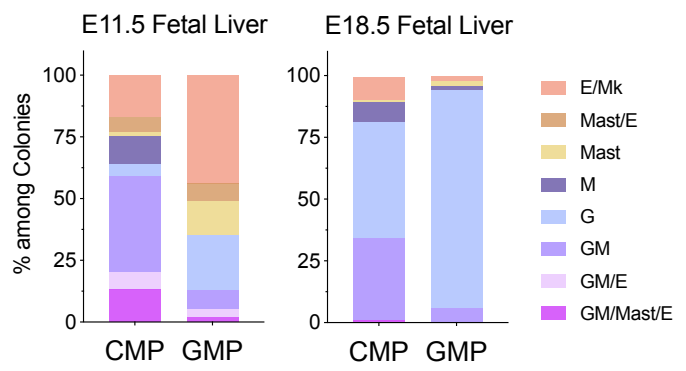

(Sorted Populations)

Fig. 4. HSC-derived and EMP-derived GMP are functionally distinct. a-d, scRNAseq analysis of E10.5 and E12.5 fetal liver EMP-derived hematopoietic progenitors (clusters 1-10, see Extended Data Fig. 3c). Index sorted cells from scRNAseq were plotted on a biexponential scale based on cell surface expression of CD16/32 and CD34. Cells from each cluster are highlighted (blue) (a). Proportions of clusters among CMP, GMP and MEP immunophenotypic populations (b). Expression (log2 UMI counts) of lineage defining transcription factors (KIf1, red blood cell; Cebpe, neutrophil; Gata2, mast cell) among cells from GP and Mast clusters. Boxes indicate cells with exclusive expression of one transcription factor (red, red blood cell; blue, neutrophil; yellow, mast cell) or co-expression (dark grey) (c). Quantification of the proportions of cells in each transcription factor expression category among each cluster (threshold $>1$ ) (d). The values of some proportions are written. e. Erythroid and myeloid potential as determined by single cell CFU assays of CMP and GMP that were sorted from E11.5 or E18.5 fetal livers. E11.5 CMP $n$ $=87, \mathrm{E} 11.5 \mathrm{GMP} n=235, \mathrm{E} 18.5 \mathrm{CMP} n=78, \mathrm{E} 18.5 \mathrm{GMP} n=66 . n$ represents numbers of analyzed colonies from one independent experiment per stage. $E$, erythroid; Mk, megakaryocyte; $M$, macrophage/monocyte; G, granulocyte; GM, granulocyte/macrophage/monocyte. 


\section{Extended Data Fig. 1}

a.

Gating Strategy for Analysis of Mature Hematopoietic Cells

E16.5 Fetal Liver
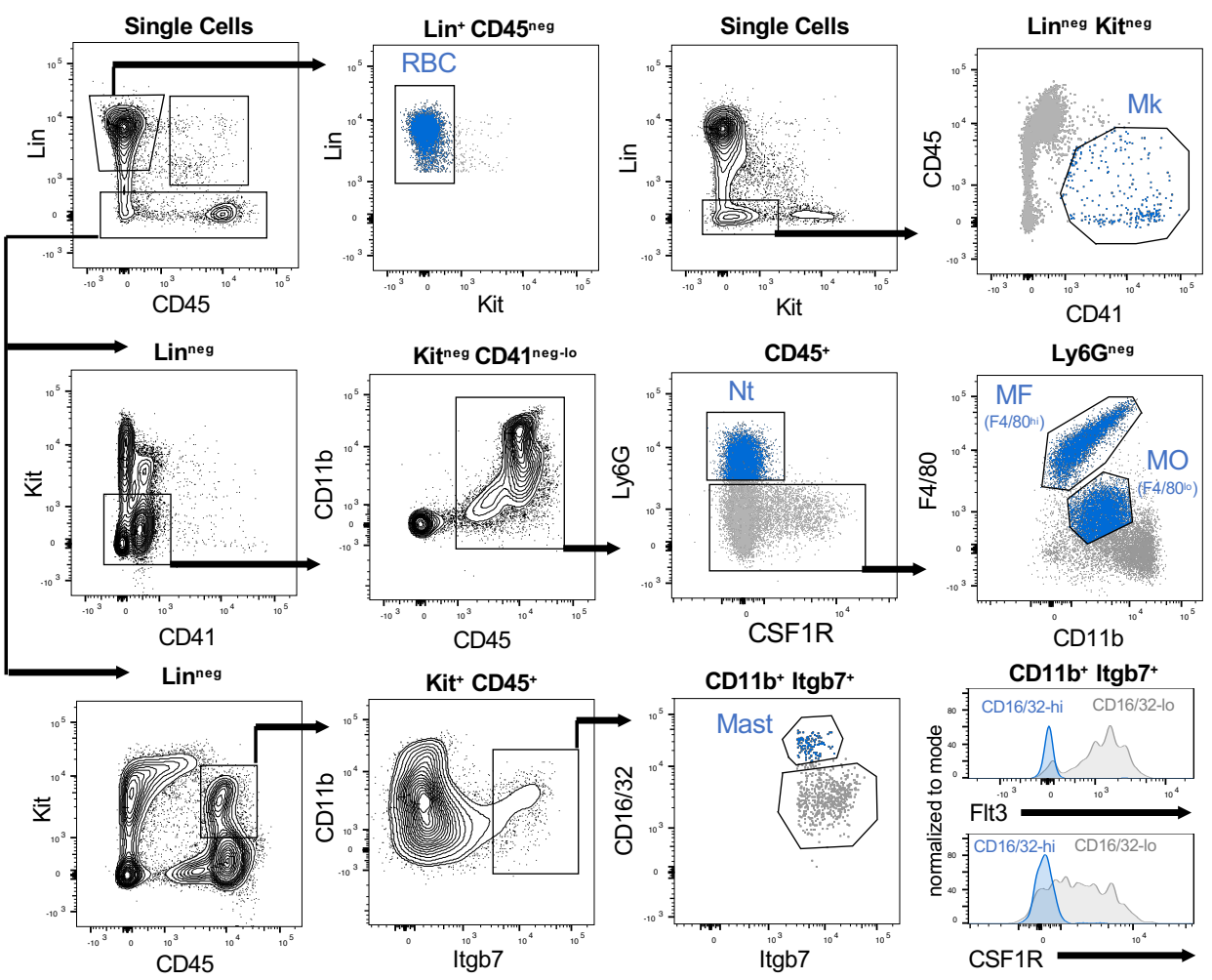

b.

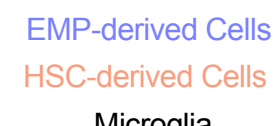
Microglia

$$
\text { Cdh5 }{ }^{\text {CreERT2 }} \text { Rosa26 } 6^{\text {YFP }}
$$

FETAL LIVER LSK

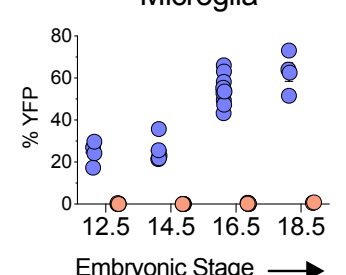

d.

$$
\text { FETAL LIVER }
$$
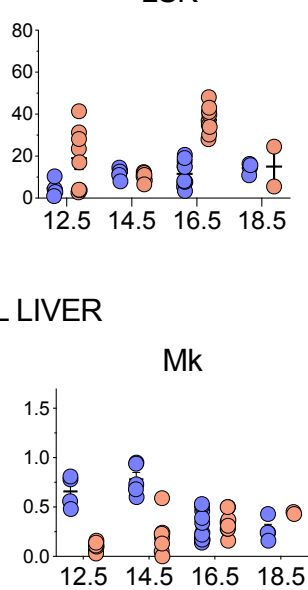

FETAL LIVER

LK

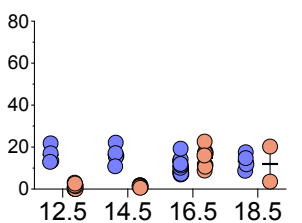

e.

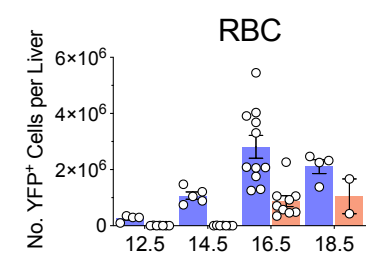

POSTNATAL (analysis at 4 weeks of age)

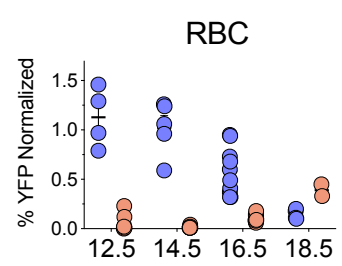

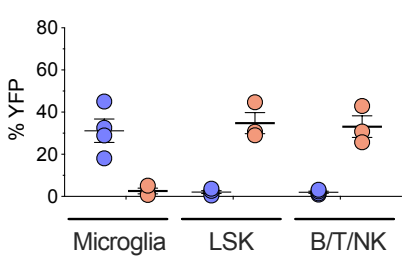

FETAL LIVER

Extended Data Fig. 1. Pulse chase labeling of mature hematopoietic cells. a, Flow cytometry gating strategy for analysis of erythroid (RBC, red blood cell; Mk, megakaryocyte) and myeloid (Nt, neutrophil; MF, macrophage; MO, monocyte; Mast, mast cell) lineages in the fetal liver. Lin (Lineage; Ter119+ CD19+ $\mathrm{CD}^{+} \mathrm{CD}^{+}{ }^{+} \mathrm{CD} 3 \mathrm{e}^{+} \mathrm{Nk} 1.1^{+}$). b-e, In vivo pulse chase labeling using Cdh5 ${ }^{\text {CreERT2 }}$ Rosa26 ${ }^{\text {YFP }}$ with E7.5 OHT injection (to label EMP-derived cells) or E10.5 OHT injection (to label HSC-derived cells). YFP labeling among microglia, LSK and LK in embryos (b). Postnatal labeling of microglia, bone marrow LSK and circulating lymphoid $\left(\mathrm{CD} 45^{+} \mathrm{Lin}^{+}\right.$and $\left.\mathrm{CD} 19^{+} / \mathrm{CD} 3 \mathrm{e}^{+} / \mathrm{Nk} 1.1^{+}\right)$cells in 4 week old mice pulsed by in utero $\mathrm{OHT}$ injection (c). YFP labeling (normalized as in Fig. 1) among fetal RBC and Mk (d). Number of YFP' cells per fetal liver (e). Numbers of biological samples are the same as in Fig. 1. Data are represented as mean \pm s.e.m. 
Extended Data Fig. 2

a.

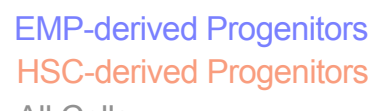
All Cells

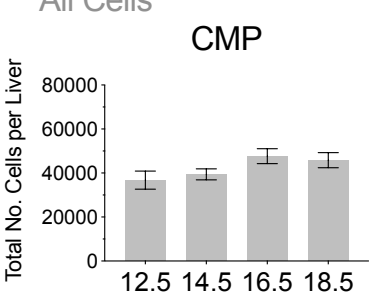

b.
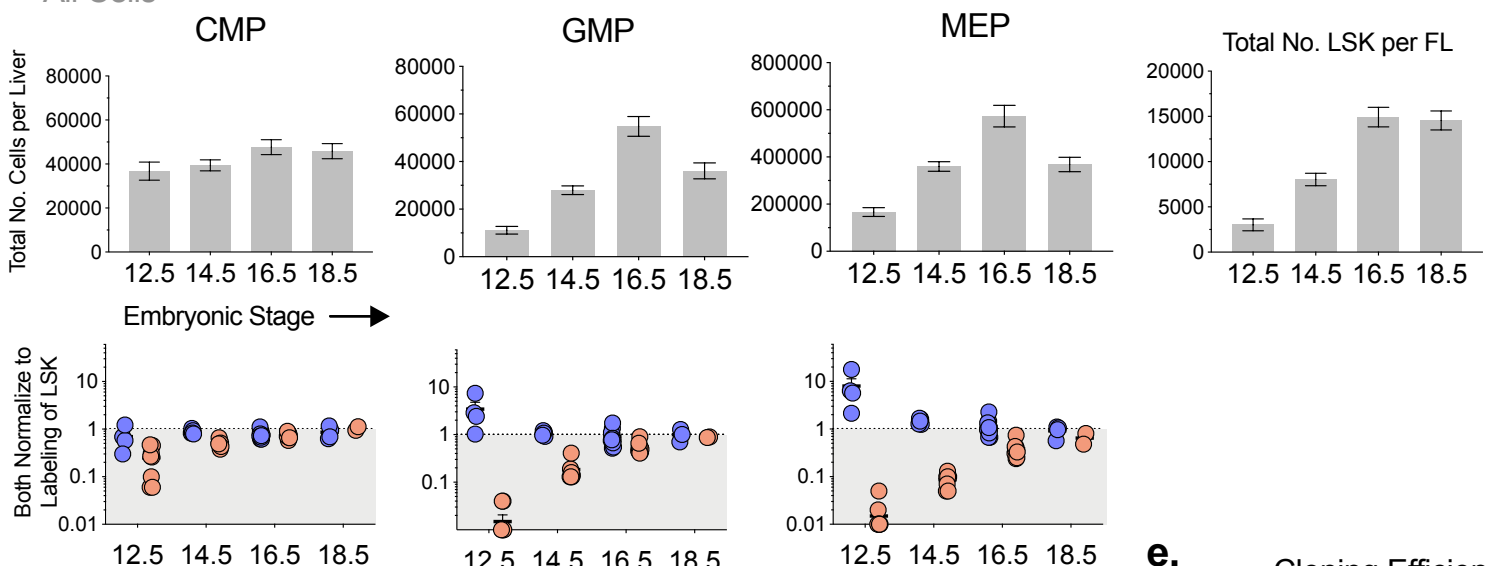

C.
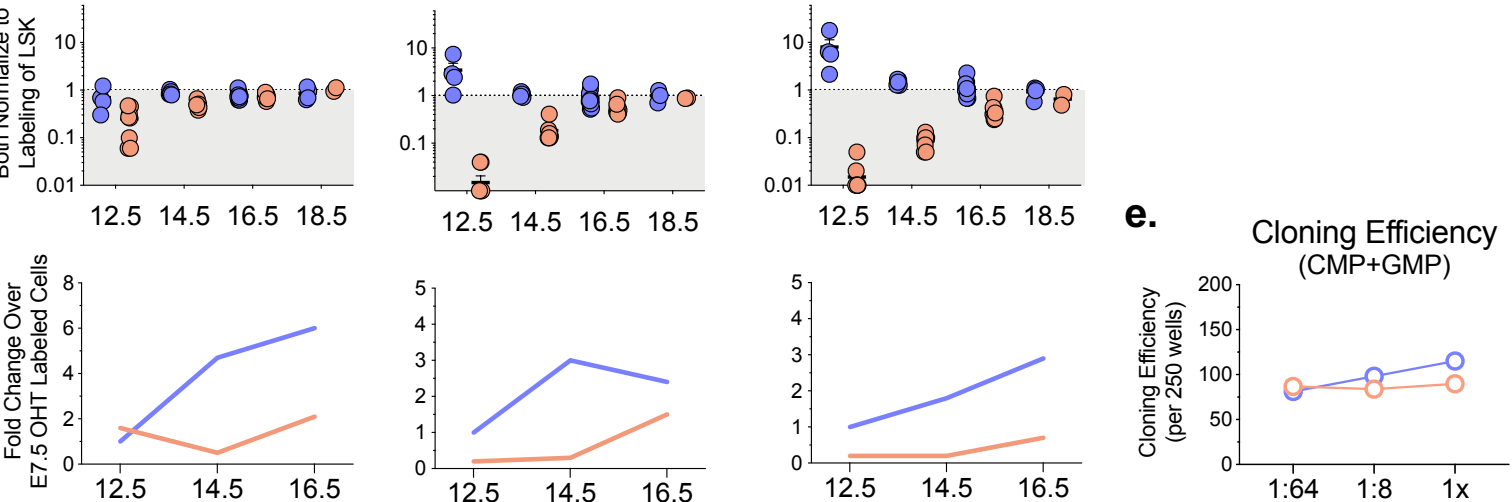

f.
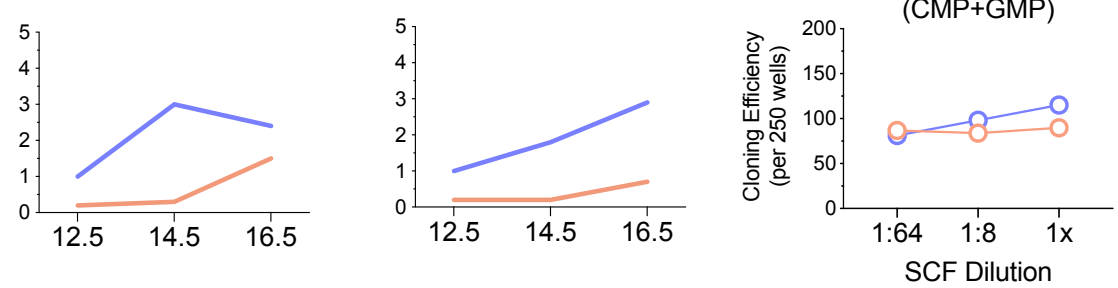$$
\text { Rosa26 } 6^{m T m G}
$$

E11.5 or E18.5 FL

$$
\text { 등 }
$$
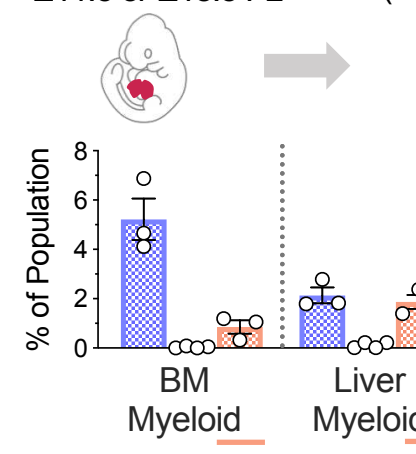

Cdh5 ${ }^{\text {CreERT2 }}$ Rosa26 ${ }^{\text {YFP }}$

FETAL LIVER

d.

.


Extended Data Fig. 3

a.

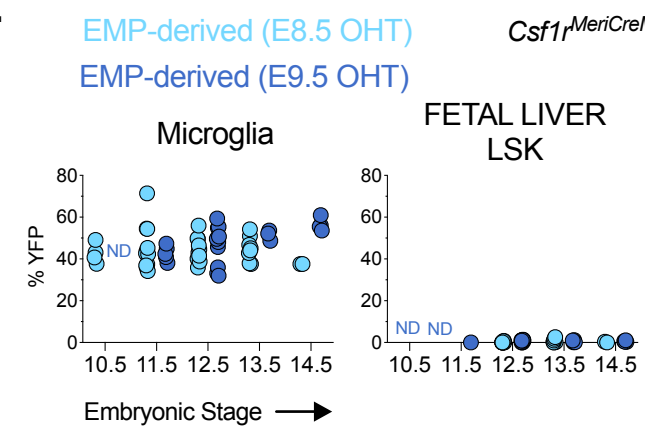

C.

$$
\begin{aligned}
& \text { Csf1 }{ }^{\text {MeriCreMer Rosa26 }} \text { YFP } \\
& \text { (E8.5 OHT > E10.5, E12.5 fetal liver LK) }
\end{aligned}
$$

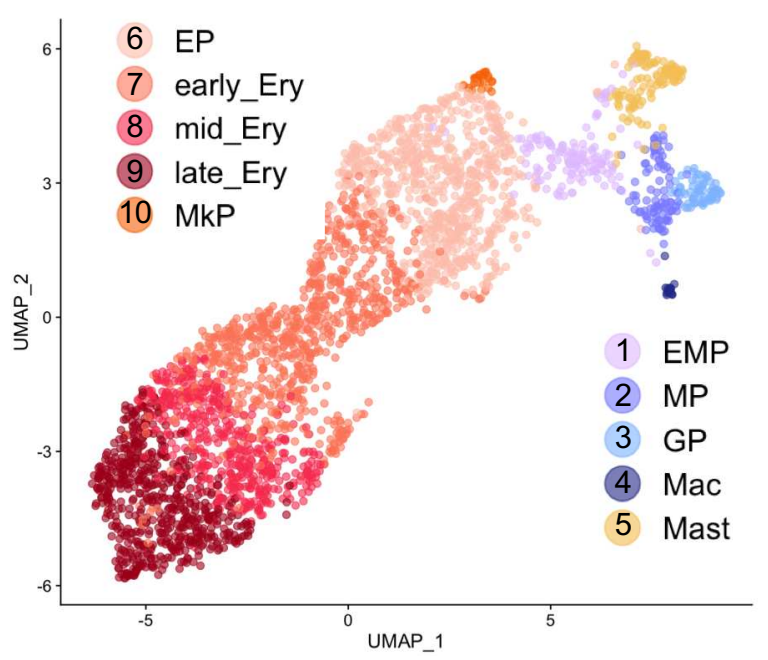

e.

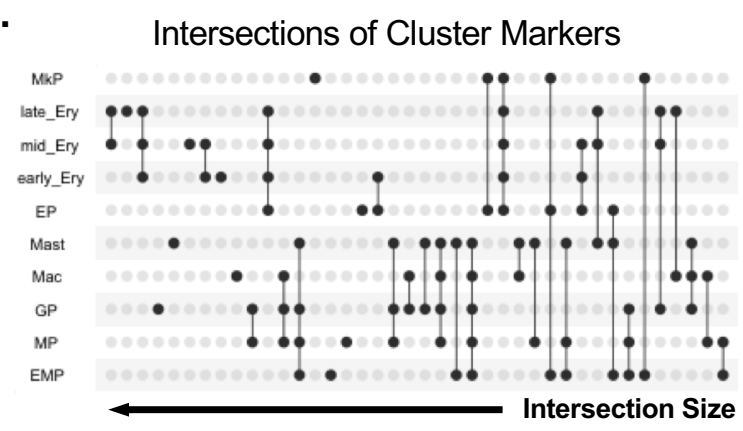

b.

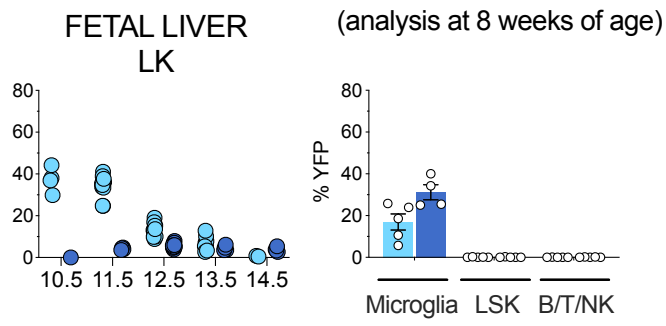

d.

Pairwise Correlation with Cells from E14 Fetal Liver (Mouse Cell Atlas)

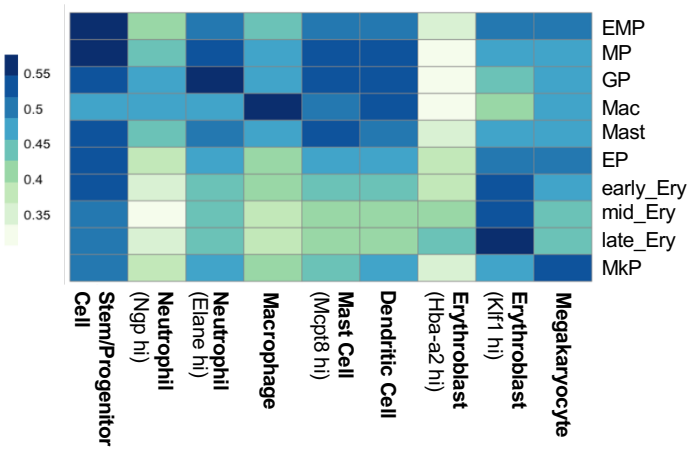

f.

Proportion of Asigned Cell Cycle Phases Among Clusters

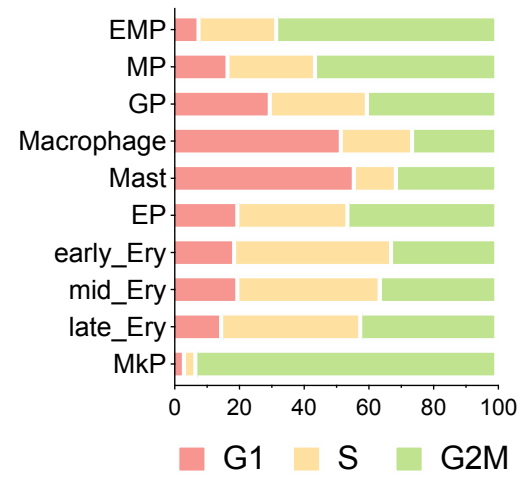

Extended Data Fig. 3. scRNAseq of EMP-derived progenitors. a-b, In vivo pulse chase labeling using Csf1 Mericremer Rosa26 YFP with E8.5 OHT or E9.5 OHT injection. YFP labeling among microglia, LSK and LK in embryos (a). Numbers of biological samples are the same as in Fig. 3. Postnatal labeling of microglia, bone marrow LSK and circulating lymphoid cells (CD45 ${ }^{+}$Lin $^{+}$and CD19+/CD3e $\left.{ }^{+} / \mathrm{Nk}^{+} 11^{+}\right)$in 8 week old mice that were pulsed by in utero OHT injection (b). E8.5 OHT $n=5$ adults; E9.5 OHT $n=5$ adults. $n$ represents biologically independent animals from one independent experiment per condition. Data are represented as mean \pm s.e.m. c-f, scRNA-seq analysis of Lin $^{\text {neg }}$ Sca1 ${ }^{\text {neg }} \mathrm{Kit}^{+} \mathrm{YFP}^{+}$fetal liver hematopoietic progenitors isolated from Csf1r MeriCreMer Rosa26 ${ }^{\text {YFP }}$ embryos pulsed with E8.5 OHT (1,590 cells from E10.5 fetal liver and 1,405 cells from E12.5 fetal liver). UMAP representation of clusters (c) and pairwise correlation with cells from the Mouse Cell Atlas (d). Intersection of cluster markers (UpsetR plot) shared between EMP and other clusters (e). Proportion of cell cycle phase assignments per cluster (f). EMP, erythromyeloid progenitor; MP, myeloid progenitor; GP, granulocyte progenitor; Mac, macrophage; EP, erythroid progenitor; Ery, erythroid; MkP megakaryocyte progenitor. 


\section{Extended Data Fig. 4}

a.

SCRNA-Seq

E10.5 \& E12.5 Fetal Liver

Proportions of Gated Cells per Cluster

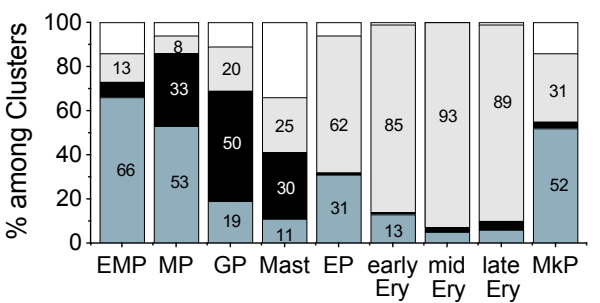

$\square$ CMP

- GMP

$\square$ MEP

$\square$ NA

d.

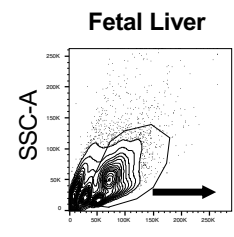

FSC-A

Live Cells

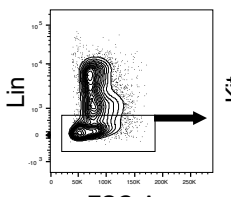

FSC-A

E11.5
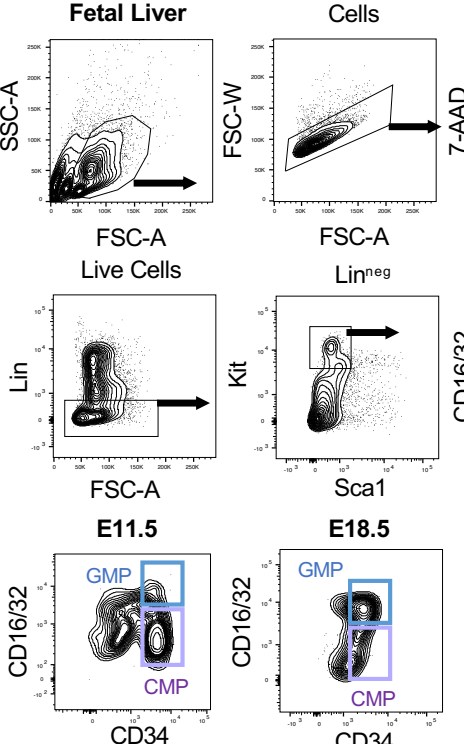

FSC-A

Linneg
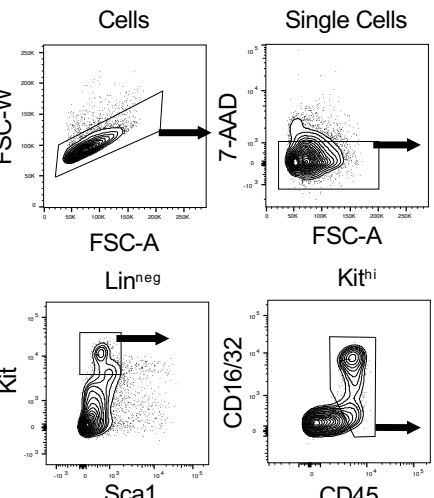

E18.5

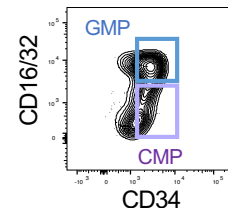

f.

CFU Assays : Colonies

\begin{tabular}{|c|c|c|}
\hline E11.5 FL & CMP & GMP \\
\hline No. Cells Sorted & 280 & 288 \\
\hline Cloning Efficiency & $1: 3$ & $1: 5$ \\
\hline Average Colony Size & $506 \pm 86$ & $189 \pm 55$ \\
\hline
\end{tabular}

b.

Paul et al., 2015

Bone Marrow Myeloid Progenitors

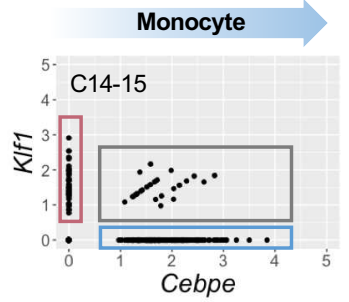

c.

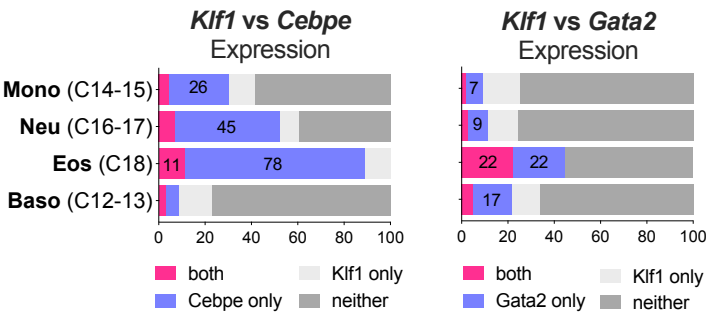

e.

CFU Assays : Day 7 Analysis

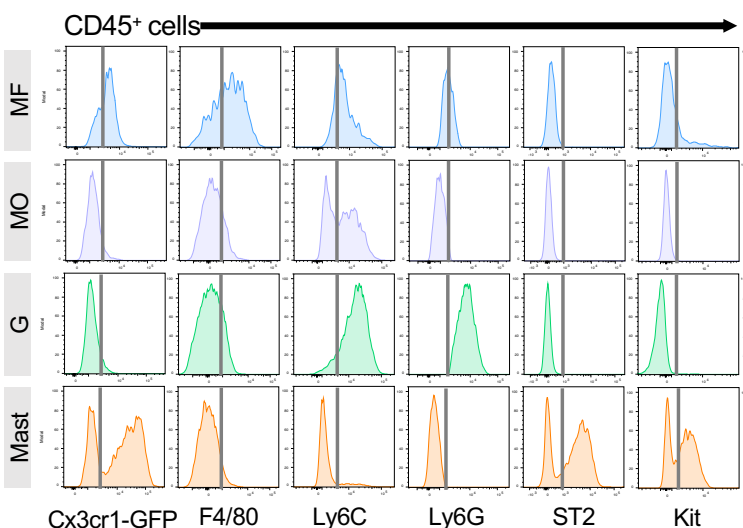

\begin{tabular}{|c|c|c|}
\hline E18.5 FL & CMP & GMP \\
\hline No. Cells Sorted & 192 & 171 \\
\hline Cloning Efficiency & $1: 2$ & $1: 3$ \\
\hline Average Colony Size & $322 \pm 46$ & $83 \pm 12$ \\
\hline
\end{tabular}

Extended Data Fig. 4. Single cell transcriptomics and colony-forming unit (CFU) assays. a, scRNAseq analysis of E10.5 and E12.5 fetal liver EMP-derived hematopoietic progenitors. Proportions of CMP, GMP and MEP immunophenotypic populations among clusters. b-c, Single cell transcriptomic analysis of bone marrow myeloid progenitors from Paul et al., 2015 with expression (log2 UMI counts) of lineage defining transcription factors (KIf1, red blood cell; Cebpe, granulocyte; Gata2, mast cell or basophil). Expression categories for Klf1 and Cebpe among subsets of monocyte (clusters C14-15) and neutrophil (clusters C16-17) progenitors (b). Boxes indicate cells with exclusive expression of one transcription factor (red, erythroid; blue, myeloid) or co-expression (dark grey) (b). Quantification of the proportion of cells in each transcription factor expression category among selected clusters C12-18 (threshold>0.5) (c). d-f, Single cell CFU assays with CMP and GMP from E11.5 and E18.5 fetal livers. Sorting strategy to exclude MEP (d). Lin (Lineage; Ter119+ CD19+ CD8+ CD4+ CD3e ${ }^{+} \mathrm{Nk}^{+} \cdot 1^{+}$). Expression of cell surface markers among myeloid colonies $\left(C D 45^{+}\right)$after 7 days of culture (e). Red blood cells and megakaryocytes were scored as CD45 neg CD71+ CD41 neg or CD45 neg CD71 neg CD41+ respectively. Summary of the number of sorted colonies, cloning efficiency and average colony size per condition (f). The mean values of some proportions are written on the graph $(\mathbf{a}, \mathbf{c})$. 